\title{
Axisymmetric convection in a cylinder
}

\author{
By C. A. JONES, D. R. MOORE AND N. O. WEISS \\ Department of Applied Mathematics and Theoretical Physics, \\ University of Cambridge
}

(Received 28 May 1975)

In three-dimensional Bénard convection regions of rising and sinking fluid are dissimilar. This geometrical effect is studied for axisymmetric convection in a Boussinesq fluid contained in a cylindrical cell with free boundaries. Near the critical Rayleigh number $R_{c}$ the solution is obtained from a perturbation expansion, valid only if both the Reynolds number and the Péclet number are small. For values of the Nusselt number $N \leqslant 2$ accurate solutions are provided by an expansion in a finite number of vertical modes. For Prandtl numbers $p<1$ the form of the solution changes at large Reynolds number and becomes independent of $p$; in the limit $p \rightarrow 0$ there is an effective critical Rayleigh number $R^{*}=1 \cdot 32 R_{c}$, which can also be derived by a perturbation procedure, and the Nusselt number is a function of the Rayleigh number only. Numerical experiments yield solutions for Rayleigh numbers $R \leqslant 100 R_{c}$ and $p \geqslant 0.01$. The results are similar to those for two-dimensional rolls and for $R \geqslant 5 R_{c}$ the Nusselt number shows only a weak dependence on $p$. For $p>1$ there is a viscous regime with $N \approx 2\left(R / R_{c}\right)^{\frac{1}{3}}$; when $R / R_{c} \gtrsim p^{\frac{3}{2}}, N$ increases more rapidly, approximately as $R^{0 \cdot 4}$. At high Rayleigh numbers a large isothermal region develops, in which the ratio of vorticity to distance from the axis is nearly constant.

\section{Introduction}

Astrophysical convection can be investigated by isolating individual features and studying them in numerical experiments. Although it is convenient to solve two-dimensional problems, stellar convection is intrinsically three-dimensional. The solar granulation, for example, shows an irregular pattern of roughly polygonal cells, each with a broad central column of rising gas surrounded by a narrow cold descending region. We can distinguish between geometrical features of the three-dimensional cells (such as the asymmetry between ascending and descending plumes) and fully three-dimensional dynamical behaviour. In this paper we study only the geometrical properties of laminar, three-dimensional cellular convection, and exclude the range of unsteady behaviour that requires a full three-dimensional solution of the governing equations.

It is generally thought that the heat flux across a convecting layer does not depend on the viscosity when the Prandtl number $p$ is sufficiently small and hence that the Nusselt number $N$ must depend on the product of $p$ and the Rayleigh number $R$ (Spiegel $1971 a, b$ ). No such behaviour is shown by numerical experiments on convection in two-dimensional rolls between stress-free 
boundaries, where $N$ varies approximately as $R^{\frac{1}{3}}$ and is independent of $p$ when $p \ll 1$ (Moore \& Weiss 1973). However, some model calculations using truncated equations with a fixed hexagonal planform have indicated that $N \sim(p R)^{\frac{1}{5}}$ when $p<1, p R \gg 1$ (Gough, Spiegel \& Toomre 1975). This result suggests that $N$ should be strongly dependent on $p$ in geometrically three-dimensional flows.

Rayleigh (1916) noted that we may regard the hexagon "as deviating comparatively little from the circular form". Axially symmetric convection in a cylindrical cell provides the simplest geometrically three-dimensional configuration: if fluid rises around the central axis and sinks at the perimeter there is an asymmetry between upward-and downward-moving regions. We have therefore investigated axisymmetric Rayleigh-Bénard convection in a Boussinesq fluid confined between free boundaries.

The only previous treatment of convection between free boundaries in an axisymmetric cell is due to Liang, Vidal \& Acrivos (1969), who used a perturbation expansion (Malkus \& Veronis 1958) to obtain the solution near the critical Rayleigh number $R_{c}$ and also computed one model with $R=1.06 R_{c}$. When $p \leqslant 0 \cdot 1$, their finite amplitude expansion gives

$$
N-1 \propto p^{2}\left(R-R_{c}\right) .
$$

For this problem we can calculate both a Péclet number $P e$ and a Reynolds number $R e$, based on the maximum vertical velocity. The perturbation procedure is valid only if $P e$ and $R e$ are both small: we find that, for $p \geqslant 1$, the Nusselt number is accurately estimated if $R \lesssim 1 \cdot 5 R_{c}$; but, for $p \ll 1$,

$$
R e \approx 100\left(R / R_{c}-1\right)
$$

and (1) is accurate to within $10 \%$ only for $R \lesssim 1 \cdot 1 R_{c}$.

A full solution to this problem requires a nice combination of analytical and computational techniques. Small amplitude convection can conveniently be studied by expressing each variable as a truncated Fourier series in the vertical co-ordinate. Four modes are sufficiently accurate for $N \leqslant 2$. The solutions obtained for $10^{-4} \leqslant p \leqslant 1$ agree with the perturbation expansion provided $R e \lesssim 10$. As the Reynolds number increases, advection of vorticity becomes important and the dependence of $N$ on $p$ in (1) disappears. For $p \ll 1$ the heat transfer is unaffected by convection until $R$ is increased to a value $R^{*} \approx 1 \cdot 32 R_{c}$ and thereafter

$$
N-1 \propto R-R^{*}
$$

and is independent of $p$. Moreover, the value of $R^{*}$ can be obtained from a perturbation procedure valid when $p \ll 1$.

This modal analysis provides a bridge between perturbation expansions and numerical experiments. We have computed results with $1 \cdot 1 \leqslant R / R_{c} \leqslant 100$ for $0 \cdot 01 \leqslant p \leqslant 6.8$ and also for infinite Prandtl number. At low Rayleigh numbers these confirm the behaviour described above. For $R \gtrsim 5 R_{c}$ two regimes can be distinguished. In the viscous regime, when $R / R_{c} \lesssim p^{\frac{3}{2}}$, advection of vorticity is negligible and

$$
N \approx 2\left(R / R_{c}\right)^{\frac{1}{3}}
$$


and is independent of $p$. At high Reynolds numbers, when advection dominates diffusion of vorticity, we find that

$$
N \propto R^{0 \cdot 4}
$$

(though the exponent in this power law is not accurately determined).

At high Rayleigh numbers the heat transport varies only slightly with the Prandtl number and as $p \rightarrow 0$ the Nusselt number becomes independent of $p$ for all $R>R^{*}$ : at $R=100 R_{c}$, for example, $N$ decreases by $10 \%$ as $p$ rises from 0.01 to $\infty$. Moreover, the change in $N$ is confined to the range $0 \cdot 1 \lesssim p \lesssim\left(R / R_{c}\right)^{\frac{2}{3}}$. The most interesting kinematic variable is the potential vorticity $\Omega=\omega / r$, where $\omega$ is the vorticity and $r$ the normalized distance from the axis, which is advected without change by the flow. Profiles of $\Omega$ show considerable variation with both $R$ and $p$. As the Rayleigh number is increased, a large isothermal region develops in the cell and at high Reynolds numbers $\Omega$ is constant in this toroidal region, as predicted by Batchelor (1956).

Although this cylindrical model is geometrically three-dimensional, nonlinear solutions yield a heat flux that shows little dependence on the Prandtl number. In fact, though they exhibit rather more variety, these solutions are surprisingly similar to the two-dimensional rolls described by Moore \& Weiss (1973). The constraint of axisymmetry leads to steady solutions at low Prandtl numbers, in which the convecting fluid acts as a flywheel, gaining speed each time it traverses the layer until friction is eventually sufficient to maintain an equilibrium. We deduce therefore that any theory in which $N$ is a function of the product $p R$ cannot rely on steady laminar flow, and must require time-dependent dynamical behaviour. This can only be studied in a fully three-dimensional calculation.

In $\S 2$ we first of all formulate the problem to be solved. Small amplitude solutions are described in $\S 3$. We then present the results of the numerical experiments in $\$ 4$ and discuss their properties in $\$ 5$. The finite-difference methods used are described in the appendix. Finally, we relate this model to other treatments of convection and speculate on the behaviour of turbulent convection.

Although convection between free boundaries in an axisymmetric cell has only been studied by Liang et al. (1969), much more attention has been paid to rigid boundary conditions, which can be realized in the laboratory though they are probably less relevant to stars. Linear theory has been dealt with by Pellew \& Southwell (1940), Charlson \& Sani $(1970,1971)$ and Joseph (1971). Nonlinear results for $R \leqslant 5000$ were obtained by Liang et al. (1969) and compared with experiments in a cylindrical container, using an oil with a temperature-dependent viscosity, for $R<12000$. Recently Threlfall (1975) has carried out a much more extended series of experiments using gaseous helium at $2^{\circ} \mathrm{K}$ at Rayleigh numbers up to $10^{9}$. Koschmieder $(1966,1974)$ has published striking photographs of convection in a circular container, with a cylindrical cell surrounded by annular convection rolls; however Krishnamurti (1973) found that this pattern was eventually superseded by one with rolls that were everywhere perpendicular to the boundary. 


\section{Formulation of the problem}

We consider a Boussinesq fluid with a velocity $\mathbf{u}$ and a density $\rho$ such that

$$
\nabla \cdot \mathbf{u}=0
$$

and

$$
\rho=\rho_{0}\left[1-\alpha\left(T-T_{0}\right)\right]
$$

where $\alpha$ is the coefficient of thermal expansion, $T$ the temperature and $\rho_{0}$ the density when $T=T_{0}$. Then the vorticity $\omega=\nabla \wedge \mathbf{u}$ satisfies the equation

$$
\partial \boldsymbol{\omega} / \partial t=\nabla \wedge(\mathbf{u} \wedge \boldsymbol{\omega})-\alpha \nabla T \wedge \mathbf{g}+\nu \nabla^{2} \boldsymbol{\omega},
$$

where $\mathbf{g}$ is the gravitational acceleration and $\nu$ the kinematic viscosity. In addition, from the heat flow equation,

$$
\partial T / \partial t=-\nabla \cdot(T \mathbf{u})+\kappa \nabla^{2} T
$$

where $\kappa$ is the thermal diffusivity.

For axisymmetric convection we adopt cylindrical polar co-ordinates $(r, \phi, z)$ with the $z$ axis vertical, and constrain the flow to be purely meridional and independent of the azimuth $\phi$. Then

$$
\mathbf{u}=(u, 0, w), \quad \boldsymbol{\omega}=(0, \omega, 0)
$$

and, from (5), we can introduce a Stokes stream function $\psi$ such that

$$
\mathbf{u}=\nabla \wedge\left(\frac{\psi}{r} \mathbf{e}_{\phi}\right)=\frac{1}{r}\left(-\frac{\partial \psi}{\partial z}, 0, \frac{\partial \psi}{\partial r}\right),
$$

where $\mathbf{e}_{\phi}$ is a unit vector in the $\phi$ direction. It follows from (9) and (10) that

$$
\omega=-\frac{1}{r}\left[\frac{\partial^{2} \psi}{\partial z^{2}}+r \frac{\partial}{\partial r}\left(\frac{1}{r} \frac{\partial \psi}{\partial r}\right)\right]
$$

and the vorticity equation (7) simplifies to

$$
\frac{\partial \omega}{\partial t}=-\left[\frac{\partial}{\partial r}(\omega u)+\frac{\partial}{\partial z}(\omega w)\right]-g \alpha \frac{\partial T}{\partial r}+\nu\left[\frac{\partial}{\partial r}\left\{\frac{1}{r} \frac{\partial}{\partial r}(r \omega)\right\}+\frac{\partial^{2} \omega}{\partial z^{2}}\right]
$$

The nonlinear term on the right of (12) describes the advection of vorticity. As a vortex tube is transported away from the axis it stretches and its cross-section correspondingly diminishes. In the absence of viscosity the circulation round the tube remains constant (by Kelvin's theorem) and so $\omega \propto r$. For convection at high Reynolds numbers in a layer of depth $d$ it proves convenient to use, instead of $\omega$, the quantity

$$
\Omega=\omega(d / r),
$$

which is the value the vorticity would have if a ring of fluid were transported to a standard distance $d$ from the axis. $\Omega$ is an intrinsic property of the vortex ring, and remains constant in the absence of a buoyancy torque or viscosity; by 
analogy with meteorological usage we term $\Omega$ the potential vorticity. Then (11) is replaced by

and (12) becomes

$$
\nabla \cdot\left[r^{-2} \nabla \psi\right]=-\Omega
$$

$$
\frac{\partial \Omega}{\partial t}=-\nabla \cdot(\Omega \mathbf{u})-\frac{g \alpha}{r} \frac{\partial T}{\partial r}+\nu \nabla \cdot\left[\frac{1}{r^{2}} \nabla\left(r^{2} \Omega\right)\right]
$$

which closely resembles the temperature equation (8).

As a model of convection in a polygonal cell we consider the cylindrical region $0 \leqslant r \leqslant r_{0}, 0 \leqslant z \leqslant d$, within which (8), (15) and (14) must be solved, subject to appropriate boundary conditions. For simplicity we assume that the boundaries are free. On the horizontal planes $z=0, d$ both the normal velocity and the tangential stress vanish, while the temperature is fixed: hence

$$
\left.\begin{array}{c}
\psi=0, \quad \omega=0, \quad \Omega=0 \quad(z=0, d), \\
T=T_{0} \quad(z=d), \quad T=T_{0}+\Delta T \quad(z=0) .
\end{array}\right\}
$$

On the cylindrical boundary the normal heat flux and velocity and the tangential stress all vanish, so that

$$
\psi=0, \quad \omega=0, \quad \Omega=0, \quad \partial T / \partial r=0 \quad\left(r=r_{0}\right)
$$

At the axis, symmetry requires that

$$
\psi=0, \quad \omega=0, \quad \Omega=\partial \omega / \partial r, \quad \partial T / \partial r=0 \quad(r=0) .
$$

Although the model could also describe a central cell surrounded by an arbitrary number of annular rolls, as in Koschmieder's $(1966,1974)$ experiments, we shall be concerned only with a single convection cell occupying the region $0 \leqslant r \leqslant r_{0}$. The generalization from one cell to a pattern of closely packed cells cannot be realized by an axisymmetric system.

A particular configuration is defined by three dimensionless parameters: the Rayleigh number

the Prandtl number and the dimensionless cell radius

$$
R=g \propto \Delta T d^{3} / \kappa \nu
$$

$$
p=\nu / \kappa
$$

$$
\lambda=r_{0} / d \text {. }
$$

The effectiveness of convection is measured by the ratio of the total heat flux to the flux that would have been carried in the absence of convection: the Nusselt number

$$
N=\frac{2 d}{r_{0}^{2} \kappa \Delta T} \int_{0}^{r_{0}}(w T-\kappa \partial T / \partial z) r d r
$$

For a steady state $N$ is a function of $R, p$ and $\lambda$ only.

It is sometimes convenient to express the equations in dimensionless form by setting

$$
(r, z)=d\left(r^{\prime}, z^{\prime}\right), \quad t=\left(d^{2} / \kappa\right) t^{\prime}, \quad T=T_{0}+\Delta T T^{\prime}
$$


For a steady state, (8), (15) and (14) then become, in terms of dimensionless (primed) variables,

$$
\begin{gathered}
\frac{\partial\left(\psi^{\prime}, \theta^{\prime}\right)}{\partial\left(r^{\prime}, z^{\prime}\right)}=\frac{\partial \psi^{\prime}}{\partial r^{\prime}}+\left\{\frac{\partial}{\partial r^{\prime}}\left(r^{\prime} \frac{\partial \theta^{\prime}}{\partial r^{\prime}}\right)+r^{\prime} \frac{\partial^{2} \theta^{\prime}}{\partial z^{\prime 2}}\right\}, \\
\frac{1}{p} \frac{\partial\left(\psi^{\prime}, \Omega^{\prime}\right)}{\partial\left(r^{\prime}, z^{\prime}\right)}=-R \frac{\partial \theta^{\prime}}{\partial r^{\prime}}+\frac{\partial}{\partial r^{\prime}}\left\{\frac{1}{r^{\prime}} \frac{\partial}{\partial r^{\prime}}\left(r^{\prime 2} \Omega^{\prime}\right)\right\}+r^{\prime} \frac{\partial^{2} \Omega^{\prime}}{\partial z^{\prime 2}}, \\
r^{\prime} \frac{\partial}{\partial r^{\prime}}\left(\frac{1}{r^{\prime}} \frac{\partial \psi^{\prime}}{\partial r^{\prime}}\right)+\frac{\partial^{2} \psi^{\prime}}{\partial z^{\prime 2}}=-r^{\prime 2} \Omega^{\prime},
\end{gathered}
$$

where

$$
\psi=\kappa d \psi^{\prime}, \quad \Omega=\left(\kappa / d^{3}\right) \Omega^{\prime}, \quad \theta^{\prime}=T^{\prime}-(1-z) .
$$

With this scaling the Prandtl number appears only in the nonlinear term on the left-hand side of $(25)$. For $p \ll 1$ this term dominates the equation. However, in the limit $p \rightarrow \infty$ (or, more precisely, when $\Omega^{\prime} / p \ll 1$ ) the Jacobian in $(25)$ can be neglected. The solution of the system of equations is then independent of the Prandtl number and determined solely by the Rayleigh number $R$ (and the geometrical factor $\lambda$ ). In particular, from (22), the Nusselt number $N=N(R, \lambda)$ only. For large Prandtl numbers, therefore, we expect to find a viscous regime in which viscosity affects the flow only through $R$, and $N$ does not depend on $p$.

\section{Small amplitude convection}

\subsection{Perturbation expansion for small Reynolds numbers}

Linear theory (Rayleigh 1916; Chandrasekhar 1961; Liang et al. 1969) predicts the onset of instability at $R=R_{c}=\frac{27}{4} \pi^{4}$, with $\lambda=\lambda_{c}=\left(2^{\frac{1}{2}} / \pi\right) j_{1} \doteqdot 1 \cdot 725$, where $j_{n}$ is the $n$th non-trivial zero of the Bessel function $J_{1}(x)$. For sufficiently small values of $R-R_{c}$ nonlinear solutions can be obtained by the perturbation expansion technique originated by Malkus \& Veronis (1958) and applied to axisymmetric convection by Liang et al. (1969). For Prandtl numbers of order unity steady finite amplitude solutions can be expanded in powers of a single, arbitrary perturbation parameter $\epsilon$ :

$$
\left.\begin{array}{rl}
\psi^{\prime} & =\epsilon \psi_{1}+\epsilon^{2} \psi_{2}+\ldots \\
\Omega^{\prime} & =\epsilon \Omega_{1}+\epsilon^{2} \Omega_{2}+\ldots \\
\theta^{\prime} & =\epsilon \theta_{1}+\epsilon^{2} \theta_{2}+\ldots \\
R & =R_{0}+\epsilon R_{1}+\epsilon^{2} R_{2}+\ldots
\end{array}\right\}
$$

(For the remainder of this section only we shall suppress the primes on dimensionless variables.) This procedure is valid only if $|\epsilon| \ll 1$ and $|\epsilon| \ll p$. Since time is measured on the thermal scale, the Péclet number $P e \approx|\epsilon|$ while the Reynolds number $R e \approx|\epsilon| / p$, so a necessary condition for the expansion to be valid is that both the Reynolds number and the Péclet number should be small.

Substitution of (28) into (24)-(26) yields a solvability condition that determines $R_{n}$ at each order. For similar boundary conditions at $z=0,1, R_{1}=0$ and

$$
\epsilon=\left[\left(R-R_{0}\right) / R_{2}\right]^{\frac{1}{2}}
$$


With free boundaries $R_{0}=\pi^{6}\left(1+k^{2} / \pi^{2}\right)^{3} / k^{2}$ is the critical Rayleigh number for a cell of radius $\lambda=j_{1} / k$ and $R_{2}(k, p)$ depends on integrals of the first-order terms in (28). These first-order solutions are just the eigenfunctions of the linear problem, so that

$$
\left.\begin{array}{rl}
\psi_{1} & =r J_{1}(k r) \sin \pi z \\
\Omega_{1} & =\left[\left(k^{2}+\pi^{2}\right) / r\right] J_{1}(k r) \sin \pi z \\
\theta_{1} & =\left[k /\left(k^{2}+\pi^{2}\right)\right] J_{0}(k r) \sin \pi z .
\end{array}\right\}
$$

Hence the Nusselt number is

$$
N=1+\frac{2}{\lambda^{2}} \int_{0}^{1} \int_{0}^{\lambda} w \theta r d r d z=1+\frac{\epsilon^{2} k^{2}}{2\left(k^{2}+\pi^{2}\right)} J_{0}^{2}\left(j_{1}\right)+O\left(\epsilon^{3}\right) .
$$

If we restrict our attention to cells with the critical radius $\lambda=\lambda_{c}$ then $R_{0}=R_{c}$, $k^{2}=\frac{1}{2} \pi^{2}$ and, from (29) and (31),

$$
N-1=\frac{\left(R-R_{c}\right) \cdot J_{0}^{2}\left(j_{1}\right)}{6 R_{2}}=\chi\left(R / R_{c}-1\right) .
$$

The second-order perturbation expansion therefore predicts that $N-1$, the normalized convective heat transport, is proportional to $R / R_{c}-1$. The constant of proportionality $\chi$ depends only on $R_{2}$. We have computed $R_{2}$ for $p \geqslant 10^{-4}$ and find that $R_{2} \propto 1 / p^{2}$ for $p \ll 1$; as $p$ increases $R_{2}$ becomes independent of the Prandtl number and $R_{2} \doteqdot 12 \cdot 56$ for $p \gg 1$ (see figure 6 of Liang et al. 1969). Hence the parameter $\chi$ decreases as $p^{2}$ for small Prandtl numbers. Figure 1 shows $\chi$ as a function of $p$, together with the corresponding curve calculated for a hexagonal cell; the results for a square cell and for a two-dimensional roll (Malkus \& Veronis 1958) are also given. The infinite roll shows no dependence of $\chi$ on $p$ but the convective heat transport tends to zero at small Prandtl numbers for all geometrically three-dimensional configurations. At the other extreme, when $p$ is infinite, $\chi \doteqdot 1.42$ for a cylinder, which is only slightly less than the value of 1.45 for a hexagonal cell (Malkus \& Veronis 1958). It is likely that the range of $\chi$ for any realizable planform lies between the two extreme values, for a roll and for a cylinder. The decrease in $\chi$ at low Prandtl number is mainly associated with nonlinear advection of vorticity, represented by the Jacobian in (25). For twodimensional rolls the corresponding term is identically zero when the linear solution is substituted into it; hence $\chi$ does not depend on $p$.

A formal radius of convergence for the expansion (28) cannot readily be determined, though a necessary condition for this expansion to be valid is that both $\epsilon$ and $\epsilon / p$ should be small. In fact, when $p \gtrsim 1$ the perturbation expansion turns out to be reasonably accurate for $R_{c}<R \lesssim 1.5 R_{c}$, or $|\epsilon| \lesssim 5$. For $p \ll 1$, $(\epsilon / p)^{2}=\left(R-R_{c}\right) / p^{2} R_{2} \approx 0 \cdot 8\left(R-R_{c}\right)$ and the restriction is more stringent. The expansion remains adequate for $R_{c}<R \lesssim 1 \cdot 1 R_{c}$, or $|\epsilon| p \mid \lesssim 7$. We may define the Reynolds number

$$
R e=U / p,
$$

where $U$ is the maximum vertical velocity, which is determined by the solution. Then, for $p \ll 1$,

$$
R e=k \epsilon / p \propto\left(R-R_{c}\right)^{\frac{1}{2}}
$$




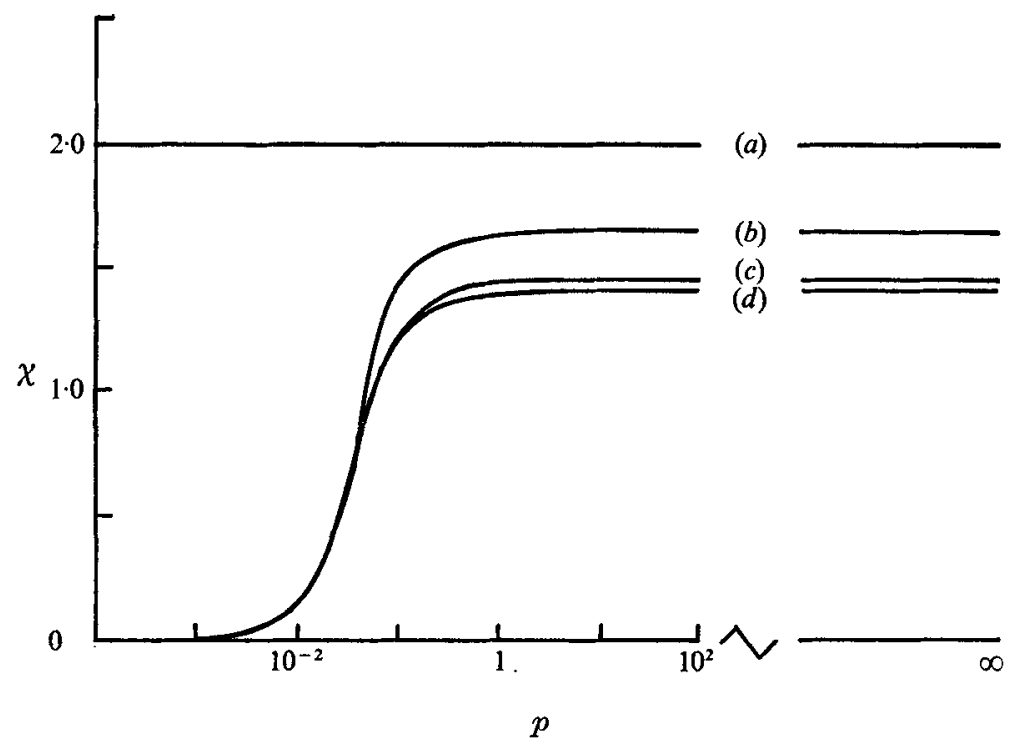

Figure 1. Convective heat transport near the critical Rayleigh number. $\chi(p)$ determined from the second-order perturbation expansion for $(a)$ two-dimensional rolls, $(b)$ square cells, (c) hexagonal cells and (d) cylindrical cells. For $p \ll 1, \chi \propto p^{2}$ for squares, hexagons and cylinders.

since $\epsilon^{2} \propto R_{\mathbf{2}}^{-1} \propto p^{2}$, and so $R e$ is independent of the Prandtl number. Advection of vorticity becomes significant when $R e \sim 10$; using values of $\chi$ computed from (32) we find, for small $p$, that $R e=10$ when $R \approx 1 \cdot 1 R_{c}$. So we would expect the perturbation expansion (28) to converge only when $\left(R / R_{c}\right)-1 \lesssim 0 \cdot 1$.

The accuracy of the expansion can be checked by comparison with fully nonlinear computations. Liang et al. (1969) reported agreement at $p=10, R=700$ $(N=1 \cdot 25)$. Our numerical experiments agree with the second-order perturbation solution for $p \geqslant 1, R \leqslant 1 \cdot 5 R_{c}$ but not for smaller Prandtl numbers. In the expansion the planform of the first-order terms is constrained to be that of the linear eigenfunctions; the nonlinear term in the vorticity equation (25) then limits the solution and produces a low heat flux. The numerical results indicate that the planform changes so as to reduce this nonlinear term as the Rayleigh number is increased. This change occurs when the Reynolds number is of order unity. For $R e>10$, vorticity is advected with the flow: this changes both the form of the solution and the amount of heat transported by convection.

\subsection{Modal expansion}

For $N \lesssim 2$ the Péclet number is small and convection is too weak for thermal boundary layers to be formed. The vertical structure of the flow remains comparatively simple. To investigate the transition from small to large Reynolds numbers we have therefore developed a modal expansion in the vertical direction (cf. the expansion in horizontal modes employed by Gough et al. 1975). We suppose 


\begin{tabular}{|c|c|c|c|c|c|c|}
\hline 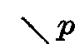 & $10^{-4}$ & $10^{-3}$ & $10^{-2}$ & $3 \times 10^{-2}$ & $10^{-1}$ & 1 \\
\hline \multicolumn{7}{|c|}{ Two modes } \\
\hline $1 \cdot 01$ & $1 \cdot 345$ & 1.345 & $1 \cdot 327$ & $1 \cdot 231$ & 1.063 & 1.008 \\
\hline $1 \cdot 02$ & 1.352 & $1 \cdot 352$ & $1 \cdot 342$ & $1 \cdot 282$ & $1 \cdot 106$ & 1.016 \\
\hline 1.05 & 1.375 & 1.375 & $1 \cdot 370$ & $1 \cdot 341$ & $1 \cdot 192$ & $1 \cdot 040$ \\
\hline $1 \cdot 10$ & $1 \cdot 414$ & $1 \cdot 414$ & $1 \cdot 411$ & $1 \cdot 394$ & $1 \cdot 282$ & $1 \cdot 081$ \\
\hline $1 \cdot 20$ & $1 \cdot 500$ & $1 \cdot 499$ & $1 \cdot 497$ & $1 \cdot 486$ & $1 \cdot 409$ & $1 \cdot 161$ \\
\hline \multicolumn{7}{|c|}{ Four modes } \\
\hline $1 \cdot 35$ & $1 \cdot 631$ & $1 \cdot 630$ & $1 \cdot 629$ & $1 \cdot 618$ & $1 \cdot 547$ & $1 \cdot 275$ \\
\hline 1.50 & $1 \cdot 784$ & $1 \cdot 783$ & $1 \cdot 783$ & $1 \cdot 773$ & $1 \cdot 710$ & $1 \cdot 406$ \\
\hline
\end{tabular}

TaBre 1. Small amplitude convection: heat flux from modal calculations. $R / R_{c}$ as a function of $N$ and $p$ for $\lambda=\lambda_{c}$

that all quantities are expanded in a Fourier series in $z$ and truncate it after the first $M$ terms. Thus we have, instead of (28),

$$
\left.\begin{array}{c}
\psi=\sum_{m=1}^{M} \Psi_{m}(r) \sin m \pi z, \\
\Omega=\sum_{m=1}^{M} \Omega_{m}(r) \sin m \pi z, \\
\theta=\sum_{m=1}^{M} \Theta_{m}(r) \sin m \pi z,
\end{array}\right\}
$$

satisfying the appropriate boundary conditions at $z=0,1$ and $r=0, \lambda_{c}$. Substitution from (35) into the time-independent equations (24)-(26) leads to a $6 \mathrm{Mth}$ order system of ordinary differential equations in the independent variable $r$, with a regular singular point at $r=0$. This system has been solved numerically for $M=2$ and 4 . In order to avoid the trivial solution $\psi=\theta=0$ it proved advisable to fix the Nusselt number as an integral constraint and then to determine the Rayleigh number as an eigenvalue. The derivatives were represented by centred second-order differences and the resulting equations solved by a NewtonRaphson procedure which iterated on the eigenfunctions and the eigenvalue simultaneously, using a program kindly supplied by Dr D. O. Gough. This method is accurate provided the Nusselt number is low enough: two modes suffice for $N \lesssim 1.3$ and four modes for $N \lesssim 2$. Moreover the computation proceeds much more rapidly than a fully two-dimensional numerical experiment.

Heat fluxes obtained from this modal expansion are shown in table 1 and figure 2, for various values of the Prandtl number and a fixed cell radius $\lambda_{c}$. For $N \leqslant 1.5$ the second-order perturbation expansion, the two-mode approximation and the fully nonlinear computations all agree, provided $p \geqslant 1$. However, for $p<1$ the perturbation expansion deviates from the other procedures except in the neighbourhood of the critical Rayleigh number, while the modal and fully nonlinear computations are in close agreement. When $p \leqslant 0 \cdot 1$ equation (32) underestimates the value of $N-1$ by $10 \%$ even for $R=1 \cdot 1 R_{c}$ : these results confirm 


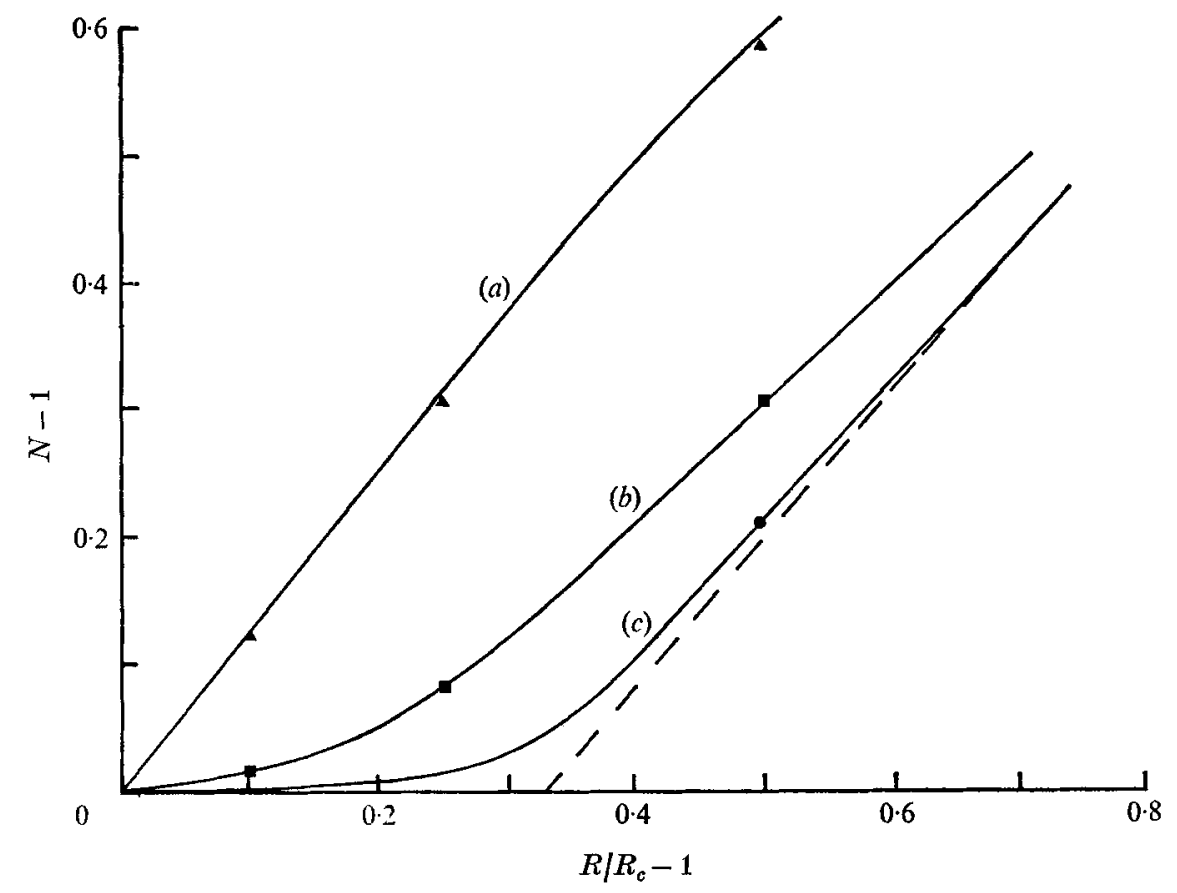

FiguRE 2. Small amplitude convection: convective heat transport as a function of Rayleigh number. Values of $N$ for several different Prandtl numbers, calculated using the modal approximation, are taken from table 1 . The full curves are for $(a) p=1,(b) p=0 \cdot 1$, (c) $p=0.03$. The dashed line is the low Prandtl number limit, given by equation (36). Also shown are results from numerical experiments, which agree with those from the modal calculations. Numerical results: $\boldsymbol{\Delta}, p=1 ; \mathbf{Q}, p=0 \cdot 1 ; \boldsymbol{O}, p=0.01$.

that the second-order perturbation expansion is reliable at low Prandtl numbers only for $R / R_{c}-1 \lesssim 0 \cdot 1$.

The modal approximation has been used to obtain results for $p=1,0 \cdot 1,0 \cdot 03$, $0.01,0.001$ and 0.0001 . Figure 2 shows that $N-1$ increases linearly at first but that for $p \leqslant 0 \cdot 1$ the slope increases when $R \gtrsim 1 \cdot 1 R_{c}$ and then settles down to a constant value, approximately equal to that for $p=1$, when $R \gtrsim 1 \cdot 4 R_{c}$. This transition from one regime to another corresponds to a change in the form of the solutions. Inspection of the fields depicted in figure 5 indicates the change in the form of $\Omega$ : the linear eigenfunction of (30) develops into a solution with $\Omega$ nearly constant along the streamlines, and the magnitude of the Jacobian

$$
\partial(\Omega, \psi) / \partial(r, z)
$$

dwindles rather rapidly as the Rayleigh number is increased.

The two-mode solutions allow us to relate changes in the potential vorticity to the Reynolds number when $p \ll 1$. For a given $R \lesssim 1 \cdot 3 R_{c}$, the value of $R e$ is fairly insensitive to changes in Prandtl number. When $R=1 \cdot 1 R_{c}, R e \approx 10$ and increases slowly such that $R e \approx 20$ at $R=1 \cdot 3 R_{c}$. The abrupt change in slope for $p \leqslant 0.01$ corresponds to $R e \approx 30$, which is typical for the change in behaviour of a passively transported scalar (cf. Weiss 1966). Thereafter, $R e$ increases steadily 

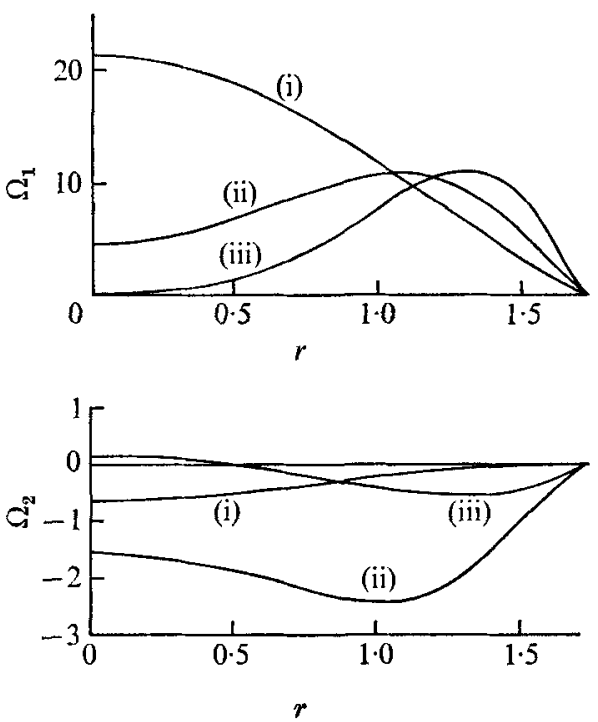

(a)

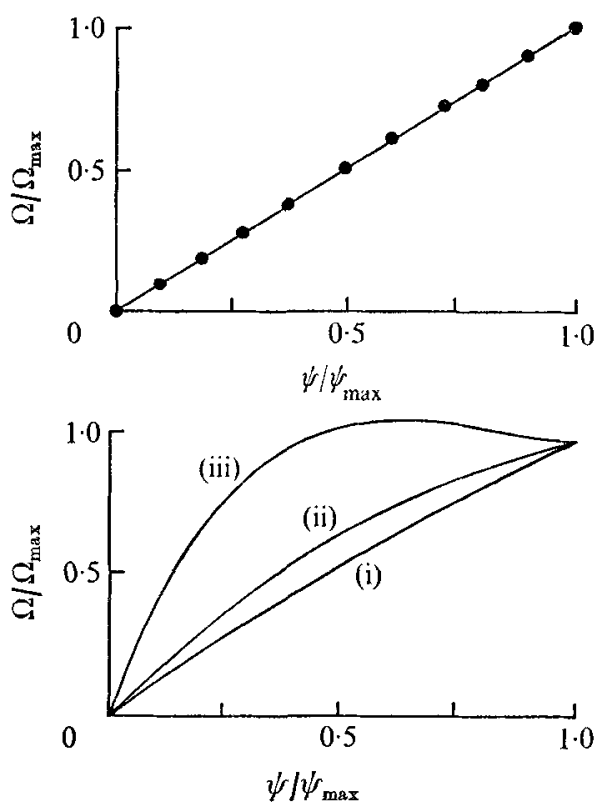

(b)

Figure 3. (a) The effect of increasing the Reynolds number on the potential vorticity $\Omega$, from the modal calculations with $M=4$. $\Omega_{1}$ and $\Omega_{2} v s$. $r$ for $N=1.05$. The three curves are for (i) $p=100, R e \doteqdot 0 \cdot 013$, (ii) $p=0 \cdot 1, R e \doteqdot 19$, (iii) $p=0.01, R e \doteqdot 252$. (b) Dependence of $\Omega$ on $\psi: \Omega / \Omega_{\max }$ vs. $\psi / \psi_{\max }$ at $z=\frac{1}{2}$, where $\psi_{\max }$ and $\Omega_{\max }$ are the maximum values of $\psi$ and $\Omega$. In the upper graph, the straight line given by the eigenvalue equation (49) is compared with results obtained using four modes, for $p=0 \cdot 01, N=1 \cdot 05$. The lower graph shows values taken from numerical experiments with $p=0.01$ and (i) $R=2 R_{\mathrm{c}}$, (ii) $R=6 R_{c}$, (iii) $R=100 R_{c}$. Apparently $\Omega$ is very nearly proportional to $\psi$ for $R^{*}<R \lesssim 2 R_{\mathrm{c}}$.

with $R$. When $R \doteqdot R_{c}$ and $R e \ll 1$, the first mode is just the eigenfunction of (30) and $\Omega_{2}$ is small. As the Reynolds number increases, $\Omega_{2}$ grows, to become comparable with $\Omega_{1}$ when $R e \approx 15$, but for $R e \gtrsim 30, \Omega_{2}$ is small again, while $\Omega_{1}$ assumes a different form. These changes are displayed in figure $3(a)$ for $N=1 \cdot 05$. At low Reynolds numbers, vorticity generated by horizontal temperature gradients is dissipated locally by viscosity. When $R e \sim 10$, advection of vorticity becomes significant and vorticity generated in rising or sinking regions is carried round along the horizontal boundaries, where it is destroyed. Hence an antisymmetric structure develops and $\Omega_{2}$ is comparable with $\Omega_{1}$. At high Reynolds numbers, vorticity is advected right round the cell, so that $\mathbf{u} . \nabla \Omega \approx 0$ and symmetry prevails again. If $\mathbf{u} . \nabla \Omega=0$ then $\Omega$ is a function of $\psi$. In fact, the results suggest that $\Omega$ is approximately proportional to $\psi$ at high Reynolds numbers, over a surprisingly wide range of Rayleigh numbers. In figure $3(b)$ values of $\Omega$ and $\psi$ at $z=\frac{1}{2}$ are plotted against each other. For $p=10^{-2}, N=1.05$ the deviation from a linear relationship is only just perceptible in the four-mode solution; even when $R=6 R_{c}$ it is only when $\psi$ is a maximum that the curve flattens out.

The $N, R$ relationships derived from table 1 show that as the Prandtl number tends to zero $N$ becomes independent of $p$ and so a limiting curve of $N$ against $R$ 
is approached in figure 2 . In this inviscid limit the Nusselt number remains unity until a second critical Rayleigh number $R^{*}>R_{c}$ is reached. More precisely, for $R_{c}<R<R^{*}$ solutions are consistent with the perturbation expansion, which predicts dimensionless velocities proportional to $p$ and $N-1 \propto p^{2}$. When $R>R^{*}$, $N$ rises linearly with a gradient similar to that predicted by (32) for $p \gtrsim 1$. Figure 2 shows a straight line fitted to the results for $p=10^{-4}$. The heat flux is given by, instead of (32), the expression

$$
N-1=\chi\left(R-R^{*}\right) / R_{c},
$$

with $\chi \doteqdot 1 \cdot 3$. The value of the second critical Rayleigh number $R^{*}$ is 1.337 as estimated from the two-mode calculation and 1.32 from the four-mode calculation. This slight difference shows that the higher modes, though small, are still present at the critical point. The existence of this critical point followed by a low Prandtl number regime in which the Nusselt number does not depend on $p$ is sufficiently important to deserve a more detailed investigation.

\subsection{The low Prandtl number limit}

As $p \rightarrow 0$ and the Reynolds number $U / p \rightarrow \infty$, the potential vorticity $\Omega$ is constrained to be almost constant along the streamlines. In this limit we can expand variables in powers of $p$, so that

$$
\psi=\sum_{n=0} \psi_{n} p^{n}, \quad \Omega=\sum_{n=0} \Omega_{n} p^{n}, \quad \theta=\sum_{n=0} \theta_{n} p^{n}
$$

The leading term in the vorticity expansion (25) is the nonlinear Jacobian: substitution from (37) gives the condition

$$
\partial\left(\psi_{0}, \Omega_{0}\right) / \partial(r, z)=0,
$$

so $\Omega_{0}$ is a function of $\psi_{0}$ and therefore constant along streamlines of the zero-order flow. The form of $\Omega_{0}\left(\psi_{0}\right)$ is determined by a balance between the generation and diffusion of vorticity across the streamlines. The right-hand side of (25) is of first order in $p$ and must be equated to the first-order Jacobians $\partial\left(\psi_{0}, \Omega_{1}\right) / \partial(r, z)$ etc. However, it is possible to avoid explicit introduction of $u_{1}$ by using the dimensionless equation of motion

$$
p^{-1}\left[\boldsymbol{\omega} \wedge \mathbf{u}+\nabla\left(\frac{1}{2} q^{2}\right)\right]+\nabla(P / \rho)=R T \mathbf{e}_{z}-\nabla \wedge \boldsymbol{\omega},
$$

where $q=|\mathbf{u}|$ and $\mathrm{e}_{z}$ is a unit vector in the $z$ direction. Integrating (39) round a closed streamline $C$, enclosing a surface $S$, yields the exact equation

$$
R \oint_{C} T \mathbf{e}_{z} \cdot d \mathbf{l}=\oint_{C} \nabla \wedge \omega \cdot d 1
$$

Following Batchelor (1956), we write the zero-order contribution to the righthand side of (40) as

$$
\begin{aligned}
\oint_{C} \nabla \wedge\left(r \Omega_{0} \mathbf{e}_{\phi}\right) \cdot d \mathbf{l} & =\oint_{C}\left[\frac{\mathbf{e}_{z}}{r} \frac{\partial}{\partial r}\left(r^{2} \Omega_{0}\right)-\mathbf{e}_{r} r \frac{\partial \Omega_{0}}{\partial z}\right] \cdot d 1 \\
& =\frac{d \Omega_{0}}{d \psi_{0}} \oint_{C} r^{2} \mathbf{u}_{0} \cdot d 1+2 \Omega_{0} \oint_{C} \mathbf{e}_{z} \cdot d 1 \\
& =\frac{d \Omega_{0}}{d \psi_{0}} \oint_{C} r^{2} \mathbf{u}_{0} \cdot d \mathbf{l},
\end{aligned}
$$


where $\mathbf{e}_{\phi}$ and $\mathbf{e}_{r}$ are unit vectors in the azimuthal and radial directions. Moreover,

Thus, from (40)-(42),

$$
\begin{aligned}
\oint_{C} r^{2} \mathbf{u}_{0} \cdot d \mathbf{l} & =\int_{S} r^{2} \omega_{0} \cdot d \mathbf{S}+\int_{S} \nabla\left(r^{2}\right) \wedge \mathbf{u}_{0} \cdot d \mathbf{S} \\
& =\int_{S} r^{3} \Omega_{0} d S-2 \iint_{S} \frac{\partial \psi}{\partial r} d r d z=\int_{S} r^{3} \Omega_{0} d S
\end{aligned}
$$

$$
\frac{d \Omega_{0}}{d \psi_{0}}=\frac{R \oint_{C} \theta_{0} \mathbf{e}_{z} \cdot d \mathbf{l}}{\oint_{C} r^{2} \mathbf{u}_{0} \cdot d \mathbf{l}}=-\frac{R \int_{S} \partial \theta_{0} / \partial r d S}{\int_{S} r^{3} \Omega_{0} d S}
$$

In particular, if the temperature is horizontally stratified, or uniform, in $S$ then $\Omega_{0}$ is constant (Batchelor 1956).

We have also, from (26), the zero-order equation

$$
r \frac{\partial}{\partial r}\left(\frac{1}{r} \frac{\partial \psi_{0}}{\partial r}\right)+\frac{\partial^{2} \psi_{0}}{\partial z^{2}}=-r^{2} \Omega_{0}\left(\psi_{0}\right)
$$

and, from (24), the zero-order heat flow equation

$$
\frac{\partial\left(\psi_{0}, \theta_{0}\right)}{\partial(r, z)}=\frac{\partial \psi_{0}}{\partial r}+r \nabla^{2} \theta_{0}
$$

Equations (43)-(45), with the boundary conditions on $\psi_{0}$ and $\theta_{0}$ and the condition $\Omega_{0}(0)=0$, define the zero-order solution. This system of equations could be solved iteratively by assuming the form of $\Omega_{0}$ and solving the inhomogeneous elliptic equation (44) for $\psi_{0}$, hence finding $\theta_{0}$ from (45) and then substituting into (43) to obtain an improved estimate of $\Omega_{0}$. Since $p$ does not appear explicitly in these equations, the solution is independent of the Prandtl number. In particular, the Nusselt number $N=N(R)$ and is independent of $p$. The smallest value of $R$ for which there exists a non-trivial solution to these equations defines the second critical Rayleigh number $R^{*}$; as $R \rightarrow R^{*}$ the left-hand side of (45) tends to zero, and $N\left(R^{*}\right)=1$.

This result suggests an interpretation of the second critical Rayleigh number $R^{*}$. Forming the scalar product of (39) with $\mathbf{u}$ and integrating over the cell allows us to express $R$ as the ratio of two power integrals:

$$
R=\int_{V} \omega^{2} d V / \int_{V} w \theta d V
$$

This forms the basis of a variational principle for the determination of $R_{c}$, the lowest Rayleigh number for which the work done by buoyancy forces can equal the viscous dissipation (Chandrasekhar 1961). At low Prandtl numbers we might minimize the same ratio, subject to the additional constraint that $\Omega=\Omega(\psi)$, to obtain another critical Rayleigh number $R^{\dagger}$ such that $R_{c}<R^{\dagger}<R^{*}$.

The approximate value of $R^{*}$ can be obtained by a procedure equivalent to taking the limit of the two-mode solution as $p \rightarrow 0$. We suppose that $\Omega_{0}$ can be expanded as a power series in $\psi_{0}$,

$$
\Omega_{0}=a\left(\psi_{0}+a_{2} \psi_{0}^{2}+a_{3} \psi_{0}^{3}+\ldots\right),
$$


where the coefficients are themselves functions of the maximum value $\Psi$ of $\left|\psi_{0}\right|$ such that $a_{r} \Psi^{r-1}=O(1)$. Now (43) can be differentiated to give

$$
\Omega_{0}^{\prime \prime} \int_{S} r^{3} \Omega_{0} d S=R \oint_{C} \frac{\partial \theta_{0} / \partial r}{r q_{0}} d l-\Omega_{0}^{\prime} \oint_{C} \frac{r^{2} \Omega_{0}}{q_{0}} d l
$$

If $C$ is the boundary $\left(\psi_{0}=0\right)$ both $\partial \theta_{0} / \partial r$ and $\Omega_{0}$ are zero; hence $\Omega_{0}^{\prime \prime}=0$ also and so $a_{2}=0$ in (47). We therefore approximate (47) by setting

$$
\Omega_{0}=a \psi_{0}
$$

so that (44) reduces to the eigenvalue equation

$$
r \frac{\partial}{\partial r}\left(\frac{1}{r} \frac{\partial \psi_{0}}{\partial r}\right)+\frac{\partial^{2} \psi_{0}}{\partial z^{2}}=-a r^{2} \psi_{0}
$$

This can be solved by separating variables to give an eigenfunction

$$
\psi_{0}=\Psi(r) \sin \pi z
$$

with an associated eigenvalue $a \doteqdot 11 \cdot 61$. (More generally, $a$ is a decreasing function of $\lambda$.) The velocity and vorticity can be derived from $\psi_{0}$ and the temperature is obtained from the linearized form of (45):

$$
\nabla^{2} \theta_{0}=-r^{-1} \partial \psi_{0} / \partial r
$$

Substitution into (46) then yields the critical Rayleigh number $R^{*}$. This procedure has been carried out by solving (50) by finite differences and then expressing $\psi_{0} / r$ as a sum of Bessel functions of the form $J_{1}\left(k_{n} r\right)$, and $\theta_{0}$ similarly in terms of $J_{0}\left(k_{n} r\right)$, so that the integrals in (46) reduce to sums. We find that $R^{*}=1 \cdot 337$, the value estimated above from the two-mode approximation.

The results of the four-mode calculation, plotted in figure $3(b)$, provide some justification for assuming that $\Omega_{0}$ is proportional to $\psi_{0}$. The consistency of this assumption can be checked by evaluating $\Omega_{0}^{\prime}$ from (43) for different values of $\psi_{0}$. These values of $\Omega_{0}^{\prime}$ vary by about $5 \%$, indicating that $a_{3} \Psi^{2} \approx 0.01$ in (47). Equation (49), though not exactly true, is a surprisingly accurate approximation in the range $R^{*}<R<2 R_{c}$. It can be shown, moreover, that this is the only possibility if $\Omega_{0}$ and $\psi_{0}$ are expanded in two vertical modes.

The results discussed so far apply only for $\lambda=\lambda_{c} \doteqdot 1 \cdot 725$. The same method can be used to find $R^{*}$ as a function of $\lambda$. As the cell radius is increased $R^{*}$ diminishes, reaching a minimum value of $1 \cdot 22 R_{c}$ when $\lambda \doteqdot 2 \cdot 9$. This behaviour is shown in figure 4 . Hence for $1.23 \leqslant R / R_{c} \leqslant 1.32$ convection is more efficient with $\lambda>\lambda_{c}$, though $N$ is a maximum for $\lambda=\lambda_{c}$ at higher Rayleigh numbers. Of course the critical Rayleigh number $R_{0}$ itself depends on $\lambda$. Indeed, $R_{0}(\lambda)>R^{*}(\lambda)$ for $\lambda \gtrsim 2 \cdot 6$; however, the cylindrical cell is by then unstable to the next mode, with an annular roll surrounding the central cell and $k \lambda=j_{2}$. (The second critical Rayleigh number for the nonlinear solution with a radial node is always greater than $R^{*}$.) In the two-mode solutions for these wide cells very little motion occurs near the axis; instead there is a rapidly circulating torus near the outer boundary. (This tendency can be seen at $R=1 \cdot 1 R_{c}$ in figure 4 .) The constraint that $\Omega$ should be constant along the streamlines is met by suppressing motion at the centre of 


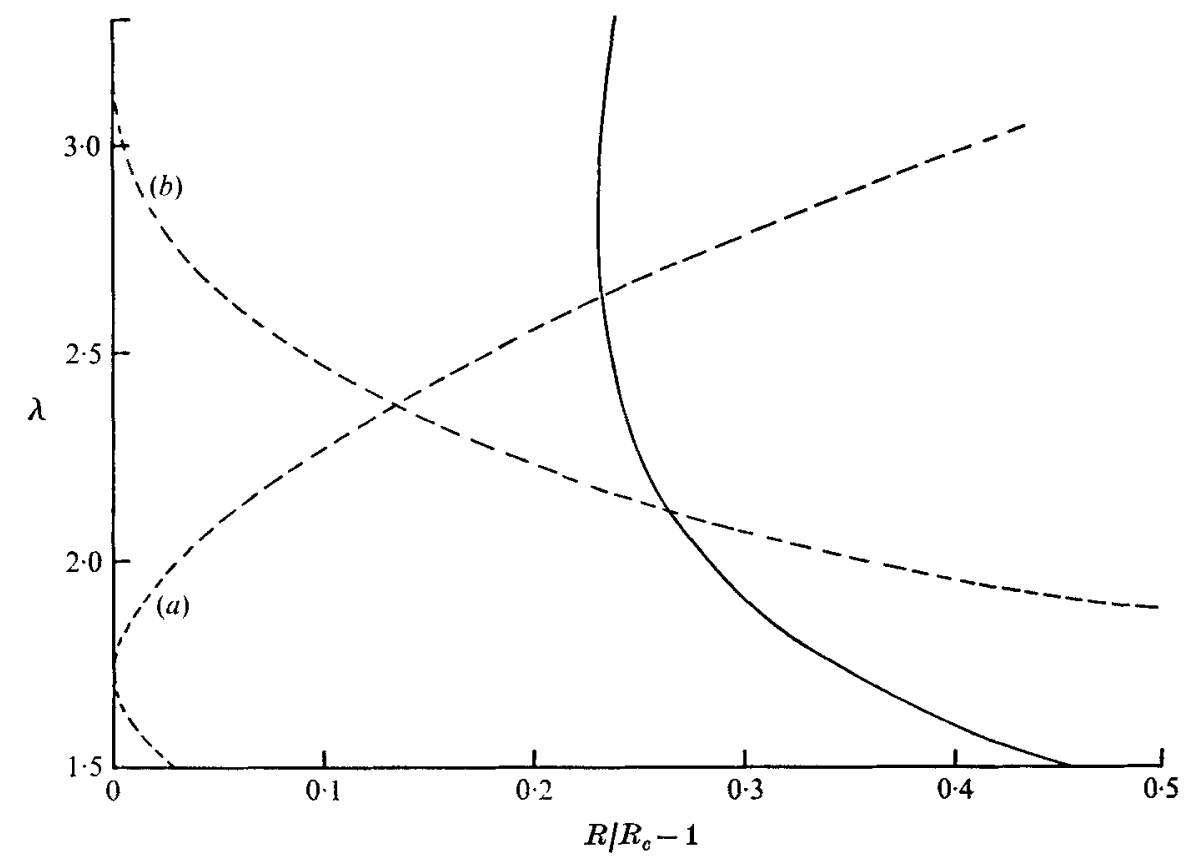

FIGURE 4. Variation with cell radius of the critical Rayleigh numbers. - - , second critical Rayleigh number $R^{*}$, which reaches a minimum for $\lambda \doteqdot 2 \cdot 9 ;---$, critical Rayleigh numbers $R_{0}$ for $(a)$ the fundamental mode and $(b)$ that with one internal node.

the cell, so that the solution resembles that for convection in a cylindrical annulus.

The models that have been described in this section show that the perturbation expansion, though it is fairly reliable at high Prandtl numbers, is valid only in the immediate vicinity of the critical Rayleigh number when $p<1$. This has been demonstrated only for cylindrical cells, though we expect the same result to hold for all geometrically three-dimensional configurations. Moreover, for convection between rigid boundaries perturbation expansions yield values of $N$ for rolls, squares and hexagons that are proportional to $p^{2}$ for $p \ll 1$ (Schlüter, Lortz \& Busse 1965). Yet Clever \& Busse (1974) have computed two-dimensional solutions which show no such decrease in the Nusselt number when $R \gtrsim 2 R_{c}$. We conjecture, therefore, that the perturbation solutions are generally misleading at low Prandtl numbers and that there will exist steady solutions for which the heat transport is only weakly dependent on $p$ at high Rayleigh numbers.

\section{Numerical experiments}

Different regimes of convection can be classified by dividing the $R, p$ plane into various regions, as shown schematically in figure 12. Linear theory yields the critical Rayleigh number; second-order perturbation theory is valid in regions I and VI, where $N-1 \propto R-R_{c}$; and the two-mode calculation of $\S 3$ is accurate in regions $\mathrm{V}$ and VI. In order to obtain solutions for $R \gtrsim 2 R_{c}(N \gtrsim 2)$ a full non- 


$\begin{array}{lclll}\qquad \backslash R_{c}^{p} & 0.01 & 0.1 & 1.0 & \infty \\ 1.1 & & & & \\ 1.25 & - & 1.018 & 1.125 & 1.152 \\ 1.5 & 1.22 & 1.086 & 1.31 & 1.34 \\ 2.0 & 1.69 & 1.31 & 1.59 & 1.64 \\ & 1.74 & 2.00 & 2.06\end{array}$

TABIw 2. Small amplitude convection: Nusselt number as a function of $R$ and $p ; \lambda=1.75$

linear computation is required. We have integrated (8), (15) and (14) numerically, using a centred second-order finite-difference scheme; the extension to cylindrical geometry of the methods used by Moore \& Weiss (1973) and described by Moore, Peckover \& Weiss (1973) is discussed in the appendix. The difference scheme has been implemented on a uniform mesh and the time-dependent equations integrated until a steady state was reached. All numerical results quoted are derived from experiments with sufficient resolution to determine the Nusselt number to an accuracy of $1 \%$ : this allows us to describe convection with $R \leqslant 100 R_{c}$ on a grid with 48 intervals vertically.

The numerical experiments can be matched to the results for small amplitude convection obtained in the last section. Values of $N$ computed on a mesh with $48 \times 84$ intervals $(\lambda=1 \cdot 75)$ are plotted in figure 2 and listed in table 2 . The results for infinite Prandtl number are obtained by reducing the parabolic differential equation (14) to an elliptic equation for $\Omega$, as described in the appendix. The analytical and numerical results are in excellent agreement for $p=0 \cdot 1,1 \cdot 0, \infty$ and $R<1.5 R_{c}$; for $p \leqslant 0.01$ computations converge very slowly and it is hard to estimate $N-1$ with sufficient accuracy at very low Rayleigh numbers. The twomode calculation is necessary to provide a bridge between numerical experiments and perturbation expansions at low Prandtl numbers.

Figure 5 shows some isotherms and streamlines, together with horizontal profiles of the potential vorticity $\Omega$ and a plot of the horizontally averaged temperature

$$
\vec{T}(z)=\frac{2}{r_{0}^{2}} \int_{0}^{r_{0}}\left(T-T_{0}\right) r d r .
$$

Here and in subsequent diagrams we constrain hot fluid to rise at the centre of the cell, although the Boussinesq equations exhibit no preference for either rising or falling fluid on the axis. At $R=1 \cdot 1 R_{c}, \bar{T}$ is scarcely affected by convection. When $p$ is infinite the motion closely resembles the linear eigenfunction of (30) and $\Omega$ is a maximum on the axis. For $p=0 \cdot 1$, however, vorticity is advected and a new maximum of $\Omega$ appears at the centre of the eddy. With $R=2 R_{c}$ this difference is even more apparent: at $p=0 \cdot 1, \Omega$ rises steeply to a maximum within the cylinder while the eddy migrates towards the outer boundary.

At low Rayleigh numbers convection is comparatively insensitive to variations in the normalized radius $\lambda$ and the maximum Nusselt number occurs for $\lambda \approx \lambda_{c} \doteqdot 1 \cdot 725$. At higher Rayleigh numbers $N$ does vary significantly with $\lambda$. Rather than explore a three-dimensional parameter space we choose the cell 


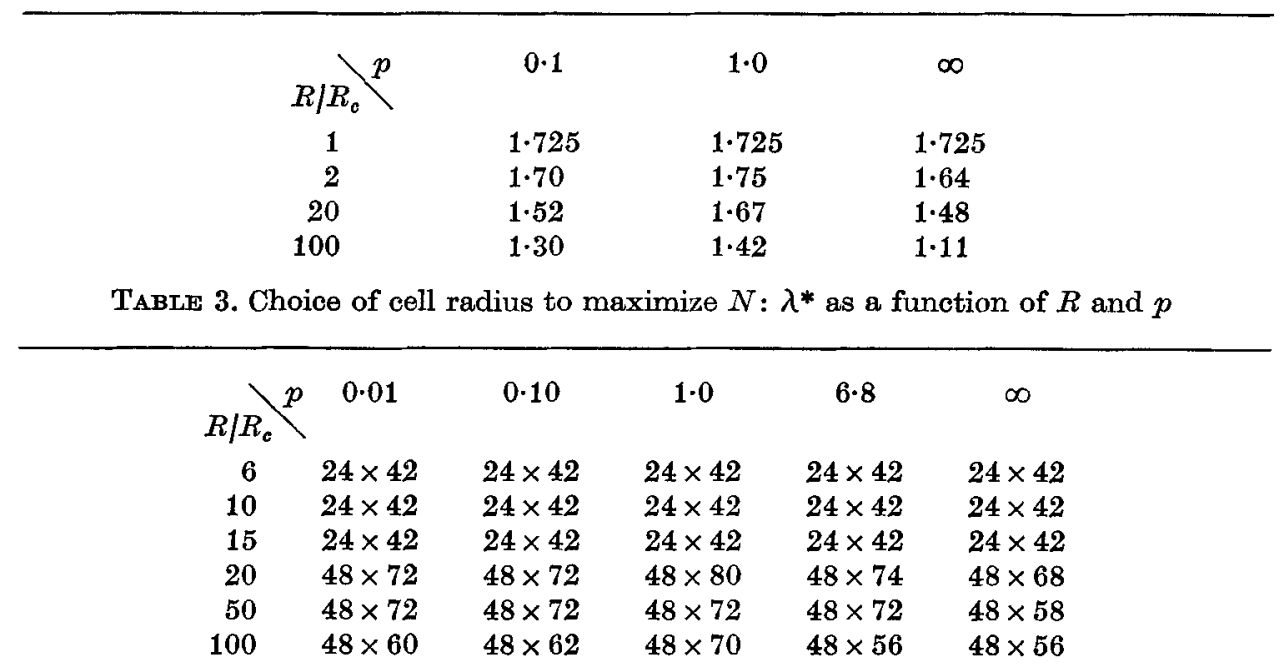

TABLE 4. Mesh sizes for numerical experiments; equal intervals in $z$ and $r$, number of vertical intervals first

width $\lambda^{*}(R, p)$ that maximizes the Nusselt number $N(R, p, \lambda)$ (Malkus 1954). Table 3 shows the variation of $\lambda^{*}$ with $R$ for $p=0 \cdot 1,1 \cdot 0$ and $\infty$. In each case $\lambda^{*}$ decreases with increasing $R$, as for two-dimensional rolls (Moore \& Weiss 1973). This variation is more marked for axisymmetric convection and it is worth noting that the values of $\lambda^{*}$ for $p=0 \cdot 1$ lie between those for $p=1 \cdot 0$ and $\infty$. However, the precise locus of $\lambda^{*}$ is probably not very significant, for the Nusselt number varies only slightly: at $R=100 R_{c}, p=\infty$, for example, $N$ changes by only $3 \%$ for a $50 \%$ increase in $\lambda$. A more important consequence of varying the cell radius is the appearance of time-dependent convection. When $p=6 \cdot 8$ finite amplitude oscillations occur at $\lambda=1.75$ for $R \geqslant 50 R_{c}$. These oscillations resemble those described by Moore \& Weiss (1973) for two-dimensional rolls; in particular, they disappear if $\lambda$ is reduced. For $R=100 R_{c}$ we have obtained steady solutions with $\lambda \leqslant 1 \cdot 17$.

The numerical experiments described below were run with normalized cell radii approximately equal to $\lambda^{*}$. The actual numbers of mesh intervals used are given in table 4 . Although 48 vertical intervals are needed to obtain an accurate estimate of the flux when $R=1 \cdot 1 R_{c}, 24$ intervals are sufficient for $2 \leqslant R / R_{c} \leqslant 10$. For higher Rayleigh numbers 48 intervals are required and these are barely adequate at $R=100 R_{c}$. The cylindrical geometry makes it difficult to extend the calculations to Nusselt numbers greater than 10.

Table 5 shows the effect on the heat flux of varying the Rayleigh number and the Prandtl number. These are the maximum values of $N$, corresponding to the normalized radius $\lambda^{*}$, obtained on the meshes given in table 4 . The values of $R$ and $p$ were chosen to allow a comparison with the corresponding Nusselt numbers for two-dimensional rolls in table 4 of Moore \& Weiss (1973); some of those results are also included here. The heat fluxes for rolls and cylinders are surprisingly similar, though $N$ is systematically lower for axisymmetric cells. Variation of $N$ with $p$ is comparatively slight but, whereas the Nusselt number 


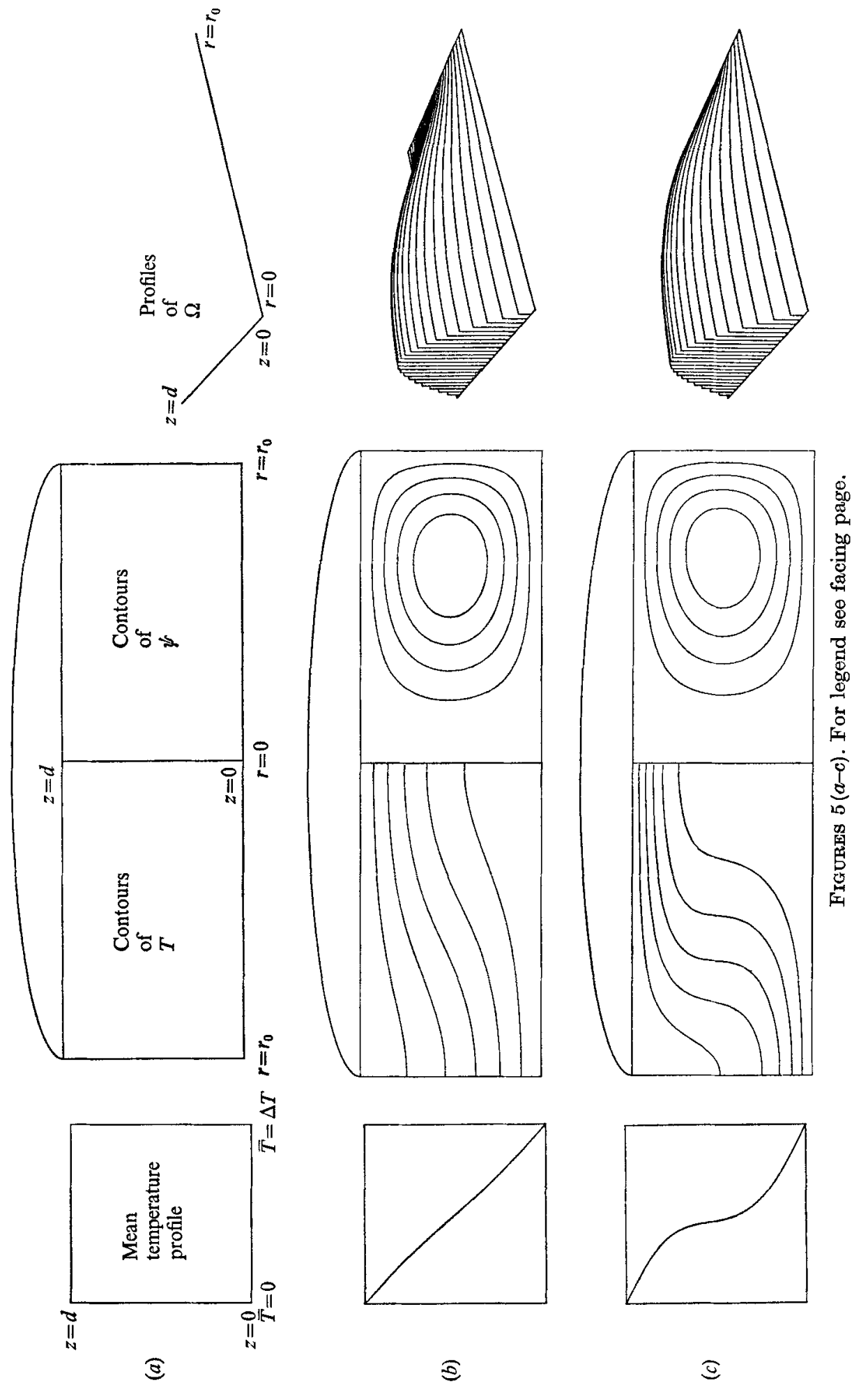



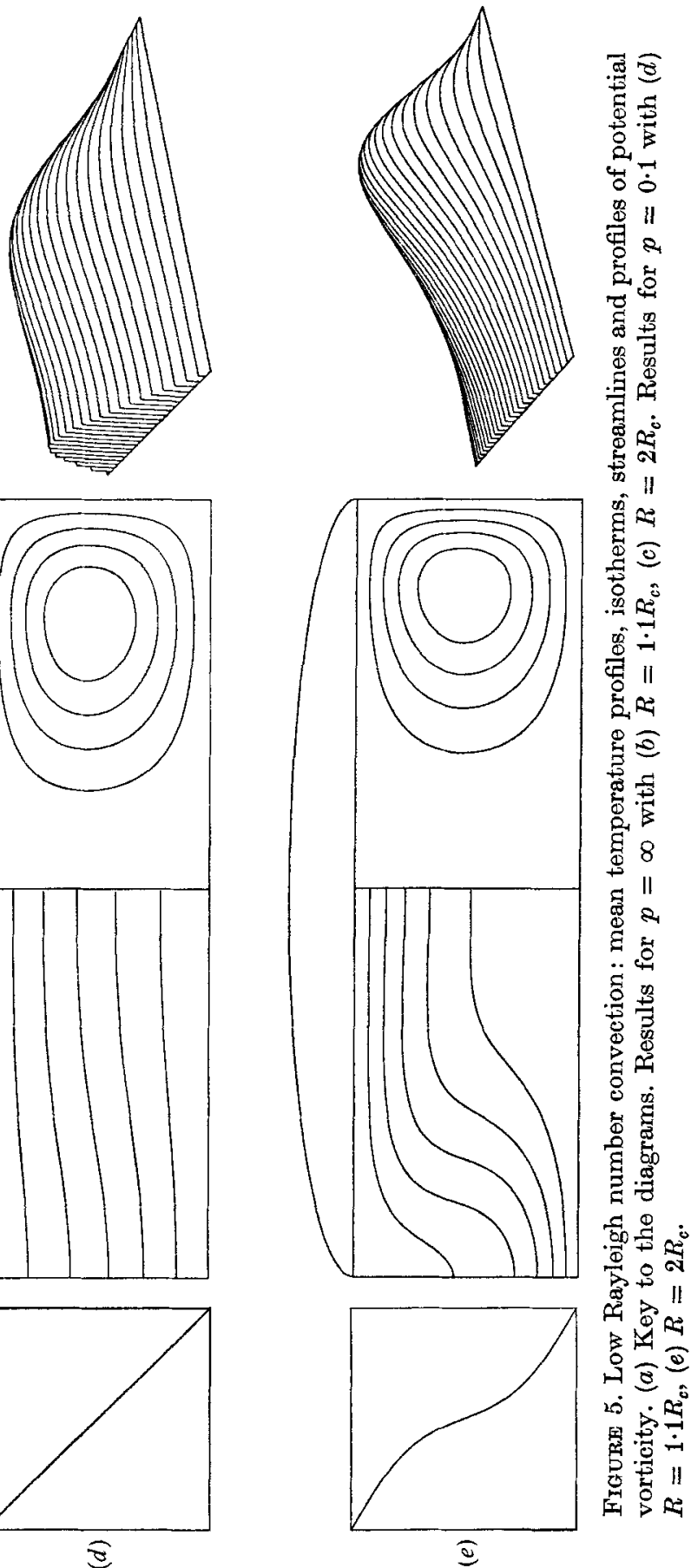


\begin{tabular}{|c|c|c|c|c|c|c|c|}
\hline \multirow{2}{*}{$R / R_{0}$} & \multicolumn{5}{|c|}{ Axisymmetric cells } & \multicolumn{2}{|c|}{ rolls } \\
\hline & 0.01 & $0 \cdot 10$ & $1 \cdot 0$ & $6 \cdot 8$ & $\infty$ & 0.1 & $\infty$ \\
\hline 6 & 3.95 & 907 & 3.49 & 3,18 & 3.55 & 3.61 & 3.55 \\
\hline 10 & $4 \cdot 04$ & $4 \cdot 06$ & $4 \cdot 17$ & $4 \cdot 16$ & $4 \cdot 18$ & $4 \cdot 39$ & $4 \cdot 24$ \\
\hline 15 & $4 \cdot 76$ & $4 \cdot 77$ & 4.82 & $4 \cdot 75$ & $4 \cdot 76$ & $5 \cdot 11$ & $4 \cdot 83$ \\
\hline 20 & $5 \cdot 44$ & 5.44 & 5.44 & $5 \cdot 31$ & $5 \cdot 34$ & $5 \cdot 67$ & $5 \cdot 37$ \\
\hline $\mathbf{5 0}$ & $7 \cdot 76$ & $7 \cdot 76$ & $7 \cdot 70$ & $7 \cdot 24$ & $7 \cdot 19$ & $7 \cdot 92$ & $7 \cdot 32$ \\
\hline 100 & 10.03 & 10.06 & $9 \cdot 96$ & 8.89 & 8.96 & $10 \cdot 18$ & 9.25 \\
\hline
\end{tabular}

Table 5. Nusselt number as a function of $R$ and $p$

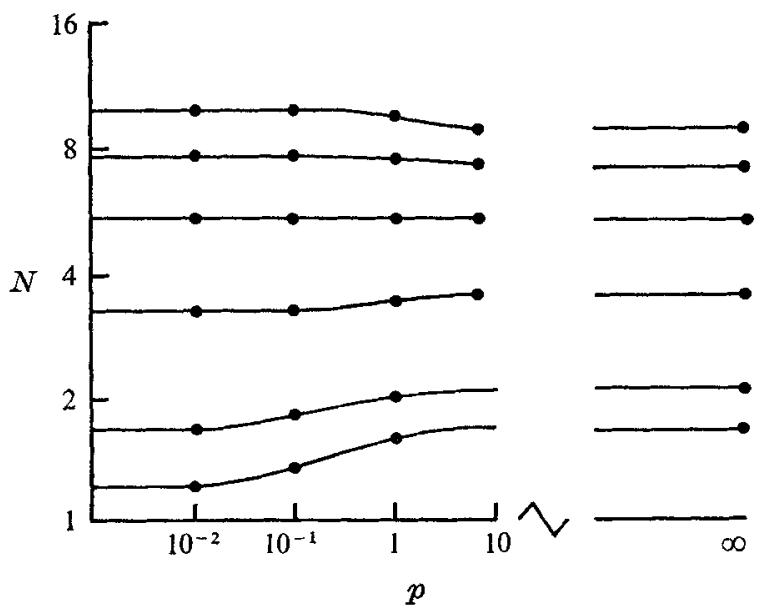

Figure 6. Nusselt number as a function of Prandtl number for (from bottom to top) $R / R_{c}=1 \cdot 5,2,6,20,50,100$. Convection becomes more efficient at low Prandtl numbers for $R \gtrsim 10 R_{\mathrm{c}}$.

for rolls always increases for $p<1$, axisymmetric cells provide more variety. At low Rayleigh numbers $N$ drops by about $6 \%$ as $p$ decreases from 10 to $0 \cdot 1$ (for rolls, $N$ rises by $0.1 \%$ over the corresponding range). This is consistent with the small amplitude results obtained above. At $R=15 R_{c}$ there is, if anything, a slight maximum at $p=1$ while for $R \geqslant 20 R_{c}, N$ increases as $p$ decreases through unity, as with two-dimensional rolls. The variation of $N$ with $p$ at different Rayleigh numbers is shown in figure 6. For a given value of $R, N$ is apparently constant both as $p \rightarrow \infty$ and as $p \rightarrow 0$, as predicted in $\S \S 2$ and 3.3 above.

For infinite Prandtl number the Nusselt number is given approximately by the power law

$$
N=1 \cdot 96\left(R / R_{c}\right)^{\frac{1}{3}}
$$

for $5<R / R_{c} \leqslant 100$. The computed results are compared with this simple power law in figure 7 . Owing to truncation errors the heat flux is slightly underestimated at high Rayleigh numbers but the results are not significantly different from the almost identical power law $N=2 \cdot 00\left(R / R_{c}\right)^{\frac{1}{3}}$ that holds for two- 


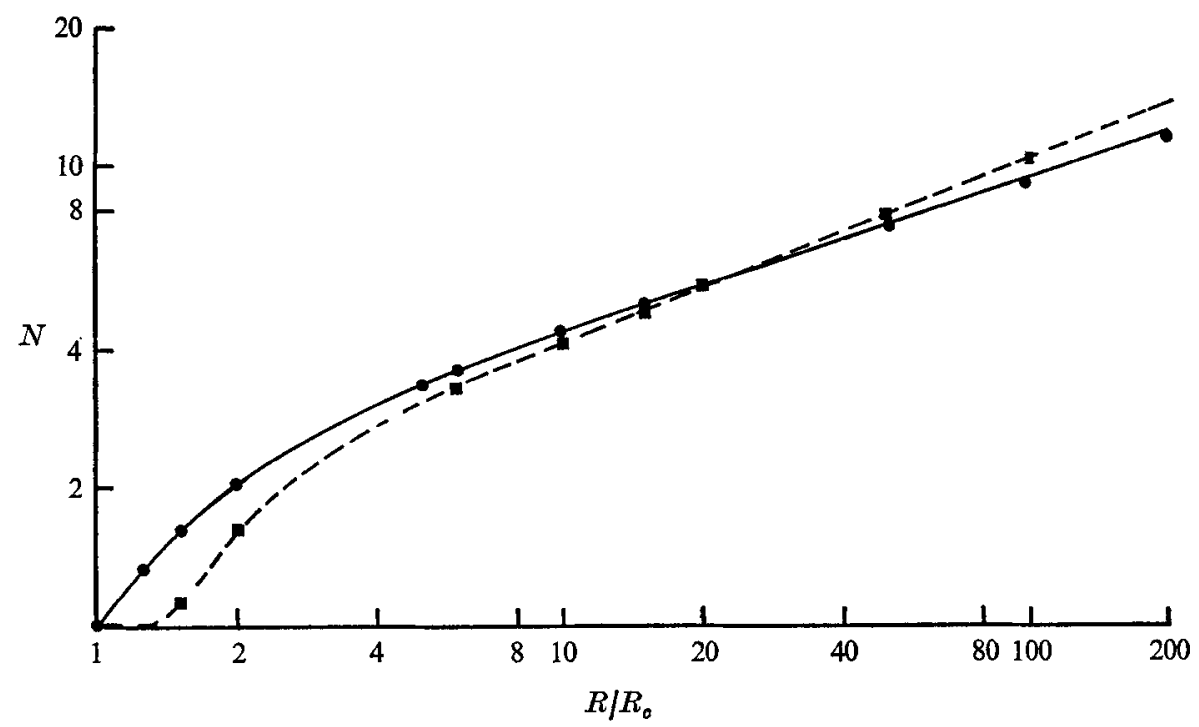

Figrre 7. Heat flux as a function of Rayleigh number. Logarithmia plot of $N$ vs. $R / R_{c}$. $\square, p=0.01 ; \bigcirc, p=\infty ;-$, equation (53) for $R \geqslant 20 R_{c} ;--_{-}$, equation (54) for $R \geqslant 20 R_{c}$.

dimensional rolls at infinite Prandtl number (Moore \& Weiss 1973). When $p=6.8$ the values of $N$ follow those for $p=\infty$ for $R \leqslant 20 R_{c}$. For $R \geqslant 50 R_{c}$, the cell radius must be reduced to eliminate finite amplitude oscillations; as a result the values of $N$ lie below those for $p=1$ or $\infty$.

At low Prandtl numbers $(p \leqslant 0 \cdot 1)$ the Nusselt number is once more a function of $R$ only. The results for $p=0.01$ are plotted in figure 7. At low Rayleigh numbers $N$ is distinctly less than for high Prandtl numbers but for $R \gtrsim 6 R_{c}$ the curve rises more steeply so as to give a larger heat transport for $R \geqslant 20 R_{c}$. It is difficult to fit an exact power law to these results, owing partly to truncation errors, but over the range $6 \leqslant R / R_{c} \leqslant 100$ the heat flux is consistent with the expression

$$
N=1 \cdot 64\left(R / R_{c}\right)^{0 \cdot 40 \pm 0.02} .
$$

The exponent apparently falls off slightly with increasing $R$ (though this is partly caused by lack of resolution) and it is conceivable that the slope is tending monotonically to some fixed value. Over this range the exponent in (37) is significantly greater than the value of 0.365 found for two-dimensional rolls (Moore \& Weiss 1973) and a fortiori greater than the slope of $\frac{1}{3}$ at high Prandtl numbers.

The qualitative behaviour of geometrically three-dimensional convection is best displayed by sets of isotherms, streamlines and profiles of the potential vorticity $\Omega$, which exhibit much more variety than the tabulated Nusselt numbers. As the Rayleigh number is increased a large isothermal region develops around the eye of the toroidal eddy. The main features to notice in figures 8-11 are the location of this region, the corresponding value of its temperature and, most striking, the variations in the potential vorticity $\Omega$. At infinite Prandtl number the eddy is fairly symmetrical and $\Omega$ has its maximum on the axis. As $R$ 

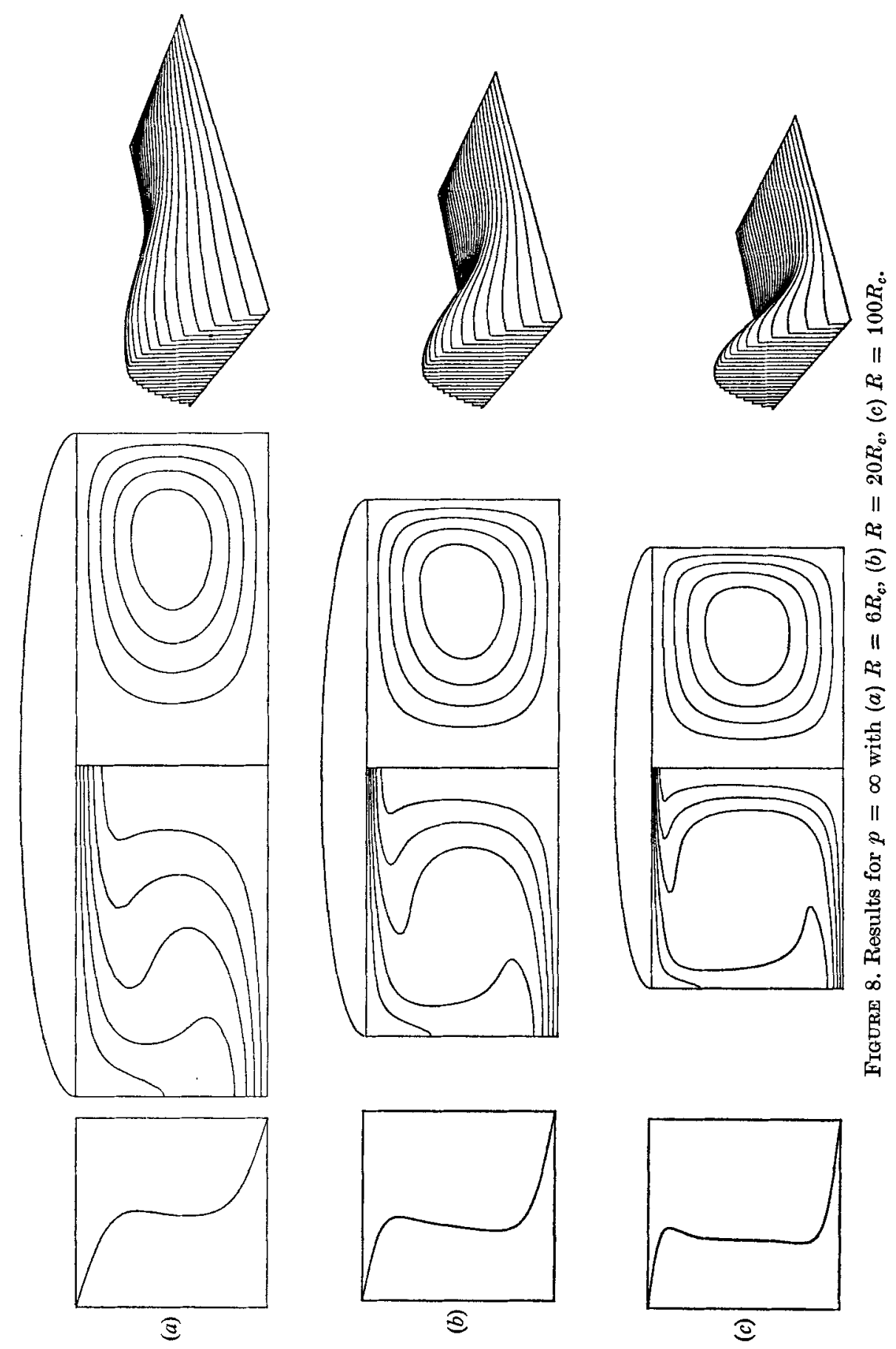

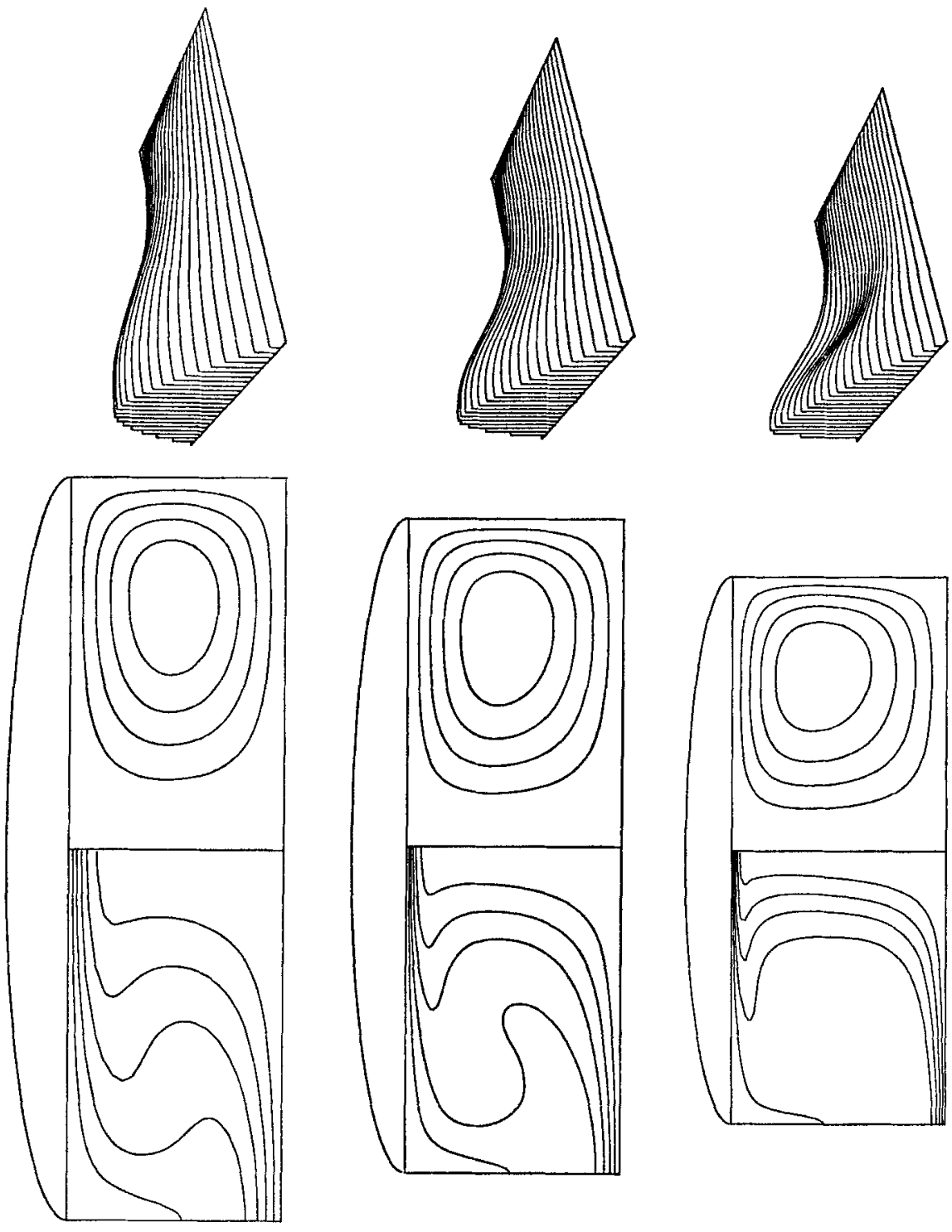

II
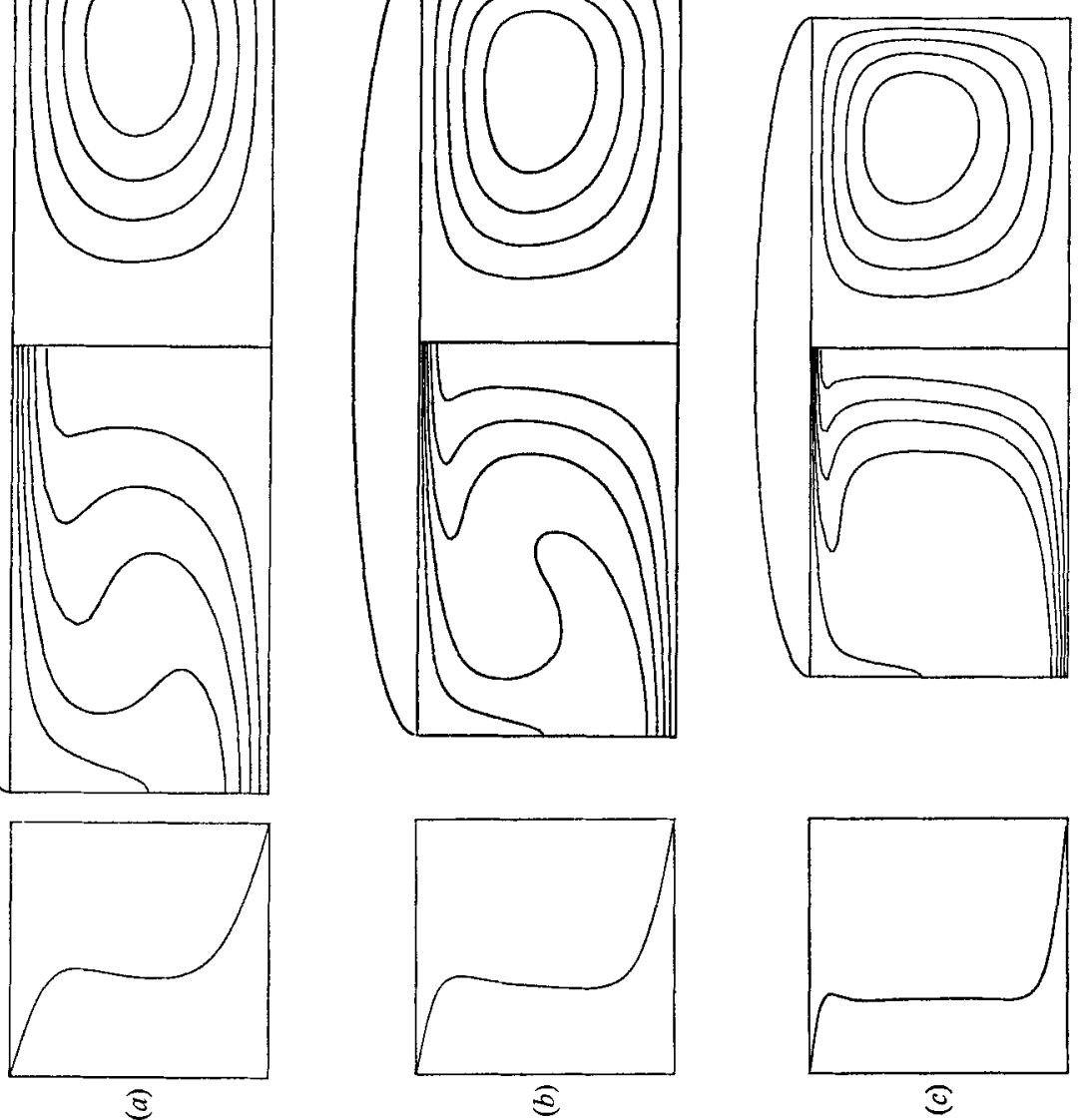

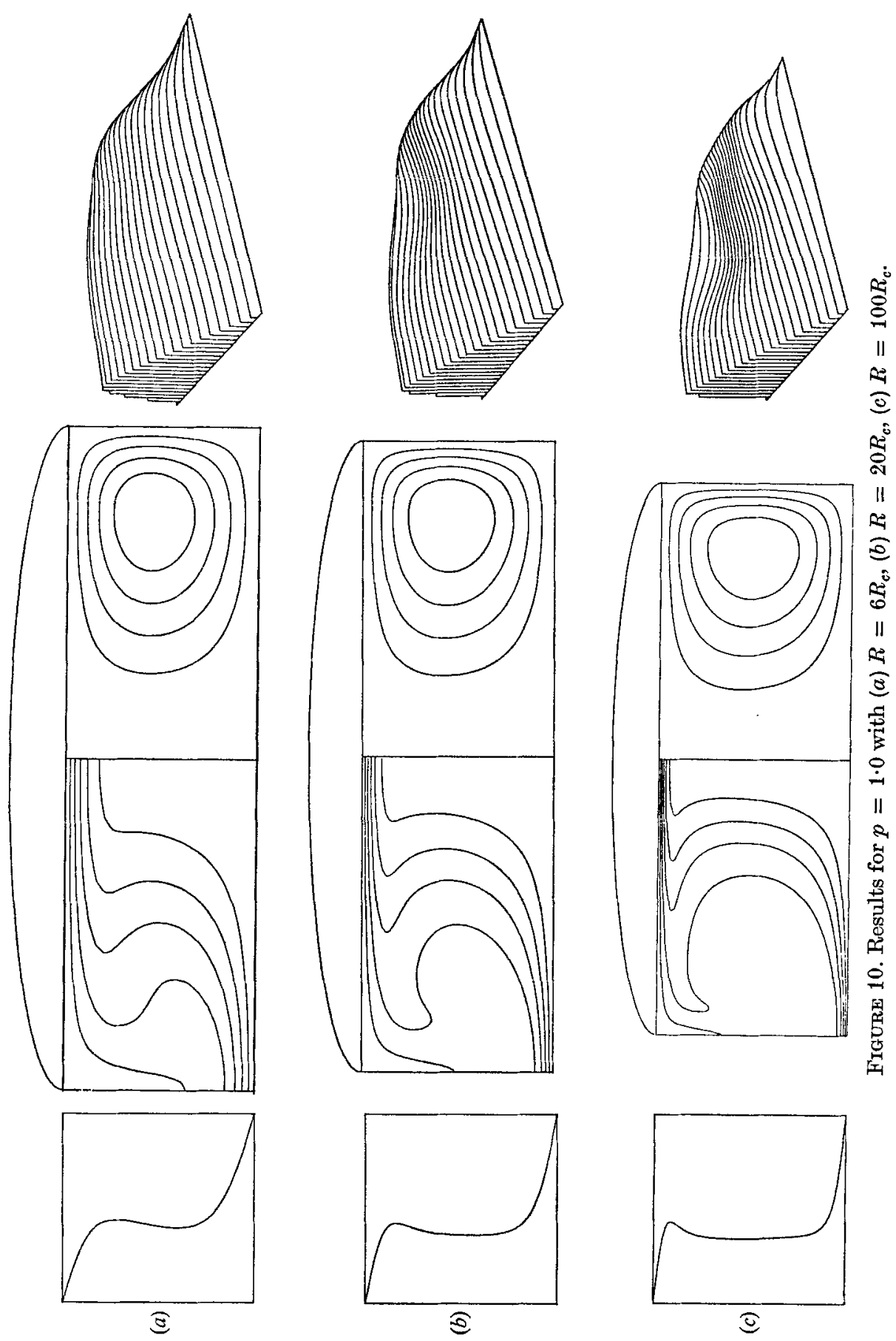

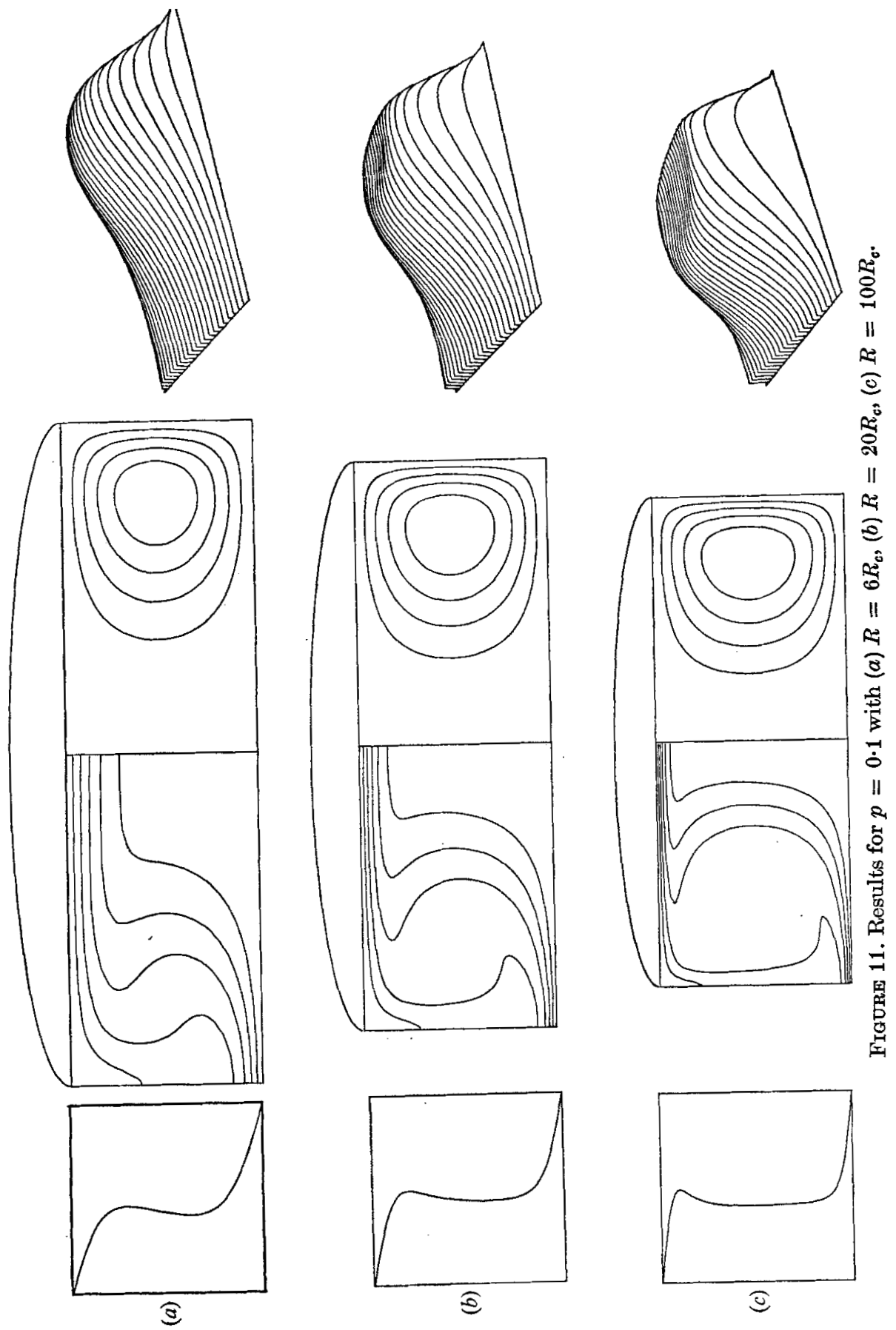


\begin{tabular}{|c|c|c|c|c|c|}
\hline$R / R_{c}$ & 0.01 & $0 \cdot 10$ & $1 \cdot 0$ & $6 \cdot 8$ & $\infty$ \\
\hline 6 & 0.507 & $0 \cdot 498$ & 0.440 & 0.443 & 0.462 \\
\hline 20 & 0.480 & 0.470 & 0.412 & 0.389 & 0.422 \\
\hline 100 & 0.440 & 0.433 & 0.383 & 0.304 & 0.389 \\
\hline
\end{tabular}

Table 6. Mean temperature of the convection cell : $\langle T\rangle \mid \Delta T$ as a function of $R$ and $p$

increases, $\Omega$ drops more steeply and the central plume plays an important role in driving the motion. The mean temperature of the layer

$$
\langle T\rangle=\frac{1}{d} \int_{0}^{d} \bar{T}(z) d z
$$

is relatively high, as shown in table 6 . When $p=6 \cdot 8$ (corresponding to water) the results are fairly similar, though $\langle T\rangle$ has decreased and $\Omega$ is less concentrated on the axis.

At low Prandtl numbers the vorticity profiles are quite different. Although $\Omega$ is not quite zero on the axis, its greatest value there (which can scarcely be seen when $p=0 \cdot 01$ ) is dwarfed by the flat maximum corresponding to the centre of the eddy, where both $T$ and $\Omega$ are uniform. The eddy itself has migrated outwards, giving a much broader central plume, though $\langle T\rangle$ is not much different from its value when $p$ is infinite. The results for $p=0 \cdot 1$ and 0.01 differ only in the magnitude of the Reynolds number, which is inversely proportional to $p$, and in small details of the flow.

The cases with $p=1$ lie between regions III and IV of figure 12 and show behaviour intermediate between the two extremes. $\Omega$ has a rather flat distribution with its maximum on the axis and at high Rayleigh numbers shows a flat depression over the centre of the eddy. The isotherms are noticeably different from those at either high or low Prandtl numbers and the mean temperature $\langle T\rangle$ is comparatively low. In all these numerical experiments the potential vorticity, which is advected with the fluid according to (15), provides the best kinematic description of the flow. The vorticity $\omega$, which drops to zero on the axis, is much less informative.

The mean temperature profiles are nearly identical at $R=6 R_{c}$, despite differences in the actual temperature distribution. Even when $R=100 R_{c}$ they show little variety, apart from the variation in $\langle T\rangle$. At high Rayleigh numbers the lack of symmetry between the central rising column of fluid and the narrow sheet descending at the perimeter affects the mean temperature. The characteristic temperature inversion appears only near the upper surface, except at infinite Prandtl number, corresponding to the spreading hot region near $z=d$. This is particularly clear when $p=1$. Away from the boundaries $d \bar{T} / d z$ is small, though the stable stratification is visible at high Prandtl numbers. 


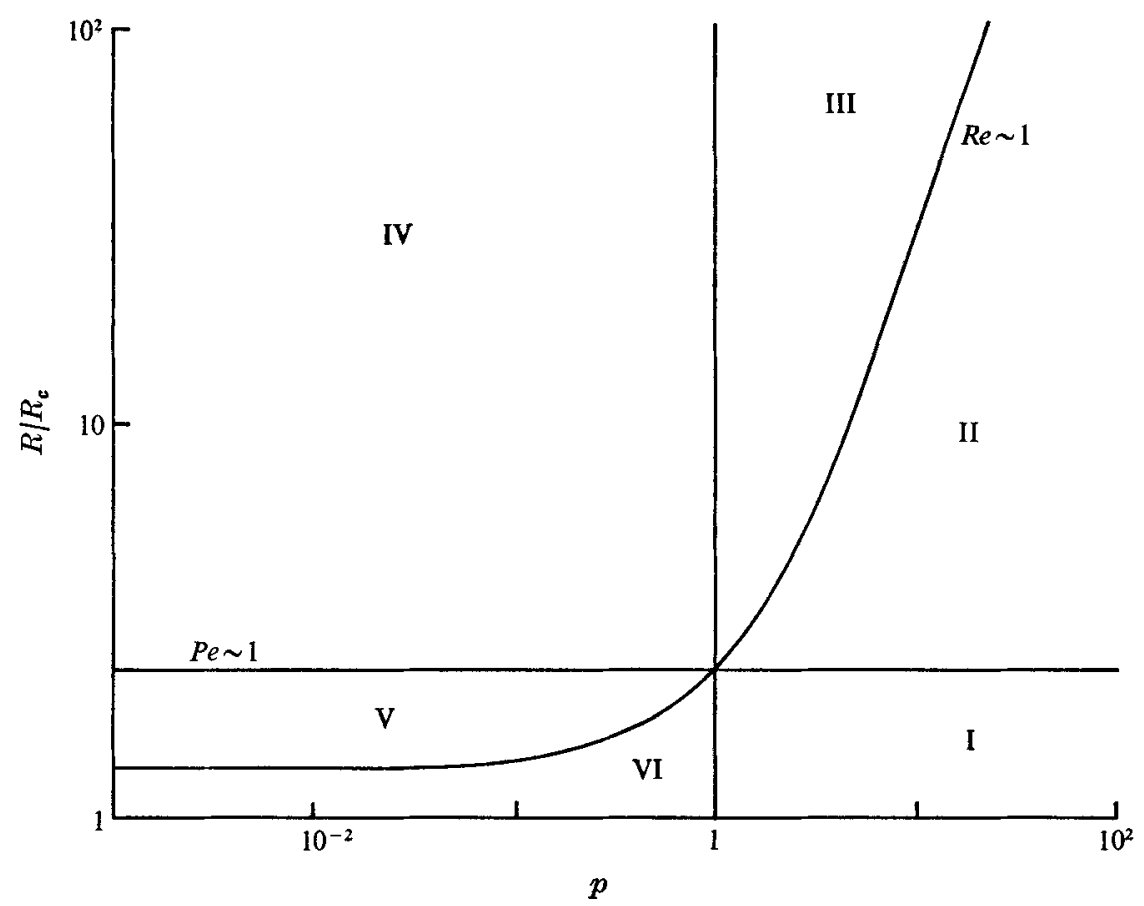

FraURe 12. Different regimes of convection. The $R, p$ plane is divided schematically into regions bounded by lines on which the Péclet number, the Reynolds number or the Prandtl number is of order unity.

\section{Discussion}

The general behaviour of axisymmetric convection cells is very similar to that of two-dimensional rolls, except in the restricted region where $R / R_{c}-1 \ll 1$ for $p \ll 1$. This behaviour can best be described by reference to figure 12 , where the $R, p$ plane is divided into six regions, separated by three lines. The horizontal line, where the Péclet number $P e=U d / \kappa \sim 1$, separates weak from strong convection; below this line, the uniformly stratified temperature distribution is merely perturbed, above it the temperature variation is concentrated into boundary layers enclosing a nearly isothermal core. The second line, where the Reynolds number $R e=U d / \nu \sim 1$, separates the viscous from the advective regime. For $p \ll 1$ this transition occurs when $R \approx R^{*}=1 \cdot 32 R_{c}$; for $p \gtrsim 1$ the change takes place when $R / R_{c} \approx p^{\frac{3}{2}}$ (ef. Moore \& Weiss 1973). Finally, high and low Prandtl number regimes are separated by the vertical line $p=1$.

For Prandtl numbers greater than unity there are three distinct regimes. At low Rayleigh numbers $\left(1 \leqslant R / R_{c} \lesssim 1 \cdot 5\right)$, in region $I$, convection is weak and the temperature field is only slightly distorted. In this region the potential vorticity $\Omega$ scarcely deviates from the linear eigenfunction of (30). Region II corresponds to a viscous regime with vigorous convection and a local balance between the generation of vorticity by the buoyancy torque and its dissipation through viscosity. Thermal boundary layers are formed, together with an isothermal zone 
that occupies most of the cell, while the Nusselt number $N \approx 2 \cdot 0\left(R / R_{c}\right)^{\frac{1}{3}}$ and is independent of $p$. The asymptotic form of the boundary layers has been investigated by Moore \& Proctor (1976). This regime includes all models with infinite Prandtl number; for finite $p$ the Reynolds number must be small, so that $1 \cdot 5 \lesssim R / R_{c} \lesssim p^{\frac{3}{2}}$ (Moore \& Weiss 1973).

At higher Rayleigh numbers, in region III, nonlinear advection of vorticity becomes important. In the isothermal zone $u . \nabla \Omega \approx 0$ and, from (43), $\Omega$ is nearly constant in a steady state (Batchelor 1956). However, viscous dissipation remains effective in the horizontal boundary layers and $\Omega$ has its maximum on the axis. We have not been able to determine the dependence of $N$ on $R$ explicitly for this regime. The behaviour of the solution is sensitive to the cell radius. For $\lambda=1 \cdot 75$, finite amplitude oscillations appear, with an amplitude that increases with $R$, as shown by the experiments with $p=6 \cdot 8$. However, these oscillations disappear when $\lambda=1$. The mechanism that produces them is the same as that described by Moore \& Weiss (1973).

When $p<1$, in region $I V$, viscosity is too weak to destroy the vorticity of a fluid element as it traverses the boundary layers. The vorticity gradually increases as fluid circulates round the cell until a balance is reached between the rate of work done by the buoyancy force and the overall viscous dissipation rate, as expressed in (46). For $p \ll 1$ the vortex ring behaves like a flywheel, circulating with a speed much greater than the reduced free-fall velocity

$$
U_{0}=(g \alpha \Delta T d)^{\frac{1}{2}}
$$

For fixed $R$, the maximum velocity $U \sim U_{0} p^{-\frac{1}{2}}$ and the Reynolds number $R e \propto p^{-1}$ : at $p=0.01, R=100 R_{c}$, for example, $U \approx 10 U_{0}$ and $R e \approx 2.5 \times 10^{4}$. In this low Prandtl number advective regime

$$
N \approx A(p)\left(R / R_{c}\right)^{0 \cdot 4},
$$

where $A$ is independent of $p$ for $p \ll 1$. We conjecture that the same power law may hold also in region III, with $A$ a slowly decreasing function of $p$, say

$$
A(p) \approx 2 p^{-0 \cdot 1} \quad(p>1) .
$$

Three-dimensional geometry introduces a qualitative difference between convection in rolls and in cylinders. For two-dimensional rolls the vorticity must be nearly constant along streamlines in the advective regime. In fact $\mathbf{u} . \nabla \omega=0$ for the linear eigenfunction and there is not too great a difference between the forms of solutions in the viscous and advective regimes. In cylindrical flow vortex lines are stretched as they move outwards from the axis and $\Omega$, rather than $\omega$, must be nearly constant on the streamlines. Unfortunately the boundary conditions on $\Omega$ and $\psi$ are dissimilar: though $\omega=0$ at all boundaries, $\Omega$ is finite on the axis. In the viscous regime the form of the solution resembles the linear eigenfunction but in the advective regime the flow adjusts its form so as to satisfy the constraint that $\mathbf{u} . \nabla \Omega \approx 0$. The profiles of $\Omega$ for $p=0 \cdot 1, R=100 R_{c}$ show that it follows the streamlines, and is nearly uniform at the centre of the eddy, as predicted by Batchelor (1956). 
In region $V$ convection is weak and no thermal boundary layers are formed, though the Reynolds number remains high (since $p<1$ ). As $R$ decreases further, the Reynolds number drops until $R e \approx 30 . \Omega$ is no longer advected round the streamlines and the Nusselt number moves off the limiting line in figure 2. For $p \ll 1$ this transition occurs when $R \approx R^{*}$ and the transition region is accessible only to the modal approximation of $\S 3$. In region VI, where, for small $p$, $1<R / R_{c} \lesssim 1 \cdot 32$, vorticity is no longer advected with the flow. The Nusselt number is small and decreases with $p$, differing thereby from region $\mathrm{I}$. As can be seen from figure 5 , the solution for $R / R_{c}=1 \cdot 1$ still shows a maximum of $\Omega$ within the cell. It is only for $R / R_{c}-1 \ll 0 \cdot 1$ that $\Omega$ resembles the linear eigenfunction; the heat transport is then given by the perturbation solution (32), with $N-1 \propto p^{2}$, but the convective transport is negligible.

\section{Conclusion}

The principal consequences of this study can be summarized quite briefly. Convection in axisymmetric cells does not differ significantly from that in twodimensional rolls except in the narrow range where both $R / R_{c}-1$ and $p$ are very small. Outside this range the heat transport shows only a weak dependence on the Prandtl number. We infer that these results would hold also for steady laminar convection in rectangular or hexagonal cells and that three-dimensional geometry alone does not have much effect.

Two-dimensional rolls are apparently unique in having linear solutions with eigenfunctions such that $\nabla \wedge(\mathbf{u} \wedge \boldsymbol{\omega})=0$. Hence the heat flux near the critical Rayleigh number is independent of $p$. In three-dimensional cells nonlinear advection of vorticity reduces the convective transport at low Prandtl numbers when $R$ is close to $R_{c}$ (as shown in figure 1). As the Reynolds number increases, the fluid acts as a flywheel, carrying vortex lines around; the planform is no longer that of the linear solutions, $\nabla \wedge(\mathbf{u} \wedge \boldsymbol{\omega})$ becomes small and $N$ ceases to depend on $p$. The effect of asymmetries between upward- and downward-moving fluid is comparatively slight.

An attempt to isolate this effect has been made by Gough et al. (1975), who studied cellular convection by solving a set of model equations in which the horizontal planform is described by a single mode. Referred to Cartesian coordinates, the vertical component of the velocity $w=f(x, y) W(z)$, where $f(x, y)$ is a prescribed function (corresponding to a separable solution of the linear problem) such that $\nabla^{2} f=-k^{2} f$, with $k$ constant. The truncated model equations include nonlinear interactions whose magnitude depends on the parameter

$$
C=\frac{1}{2} \overline{f^{3}} / \overline{f^{2}}
$$

a measure of the up-down asymmetry, where the bars denote horizontal averages. For two-dimensional rolls, for example, $C=0$, while for a cylindrical cell $f=J_{0}(\mathrm{kr})$ and $C \doteqdot 0 \cdot 176$; for rectangular cells $C=0$, again, while $C \doteqdot \mathbf{0 . 4 0 8}$ for hexagons. When $C=0$ this model reduces to the mean-field approximation; for stress-free boundaries $N$ is then proportional to $R^{\frac{1}{3}}$ and independent of $p$ as $R \rightarrow \infty$. With $C$ of 
order unity, three asymptotic regimes can be distinguished as $R \rightarrow \infty$ : for $R / R_{c} \ll p^{-1}, N-1 \sim\left(p R / R_{c}\right)^{2} ;$ for $R / R_{c} \gg p^{\frac{3}{2}} \gg\left(R / R_{c}\right)^{-\frac{3}{2}}, N \sim(p R \ln p R)^{\frac{1}{5}}$; and, for $R / R_{c} \ll p^{\frac{3}{2}}, N \sim\left(R / R_{c}\right)^{\frac{1}{3}}$. In the last case, which corresponds to the viscous regime in region II of figure 12, the asymptotic behaviour of the Nusselt number agrees with our numerical experiments. In the advective regime our results differ from the solutions to these model equations, which imply that the Nusselt number should fall off as $p \rightarrow 0$. This discrepancy is easily explained. In regions IV and $V$ of figure 12, where $R e \gg 1$, the planform of our nonlinear solutions deviates from that of the linear solution, thereby allowing $N$ to remain independent of the Prandtl number for $p \ll 1$.

Although we have obtained steady laminar solutions which are stable to axisymmetric disturbances, cylindrical convection might prove unstable to nonaxisymmetric perturbations, corresponding to the oscillatory mode for twodimensional rolls (Busse 1972). To investigate this problem requires a threedimensional calculation, which we have not attempted. Moreover, it is unrealistic to consider a cylindrical cell in total isolation. The oscillations of convection rolls set in as a collective instability, at a fixed Reynolds number of order unity (Busse 1972). They can be described in terms of the behaviour of parallel vortex tubes in a neutrally stratified, inviscid fluid (Busse 1972; Clever \& Busse 1974). At finite amplitudes viscosity, though small, must be significant, for it allows the vortex tubes to reconnect. The analogous problem for a tesselated pattern of cellular convection is that of an assemblage of closely packed interacting vortex rings. Their behaviour might provide an adequate model of turbulent convection.

The cylindrical solutions described here provide a valid description of convection between free boundaries at low and moderate Rayleigh numbers. However, steady laminar flow is unlikely to persist for arbitrarily large Reynolds numbers. At high Rayleigh numbers and low Prandtl numbers the solutions, both for rolls and for axisymmetric cells, have speeds far greater than the reduced free-fall velocity $U_{0}$. The time taken to attain a speed $U$ is greater than $\tau \sim\left(U / U_{0}\right) \tau_{0}$, where the free-fall time $\tau_{0}=d / U_{0}$. Thereafter, a steady state is approached on the viscous time scale $\tau_{\nu}=d^{2} / \nu=\left(U_{0} d / \nu\right) \tau_{0}$. If $U \gg U_{0} \gg \nu / d$ both $\tau$ and $\tau_{\nu}$ are much greater than $\tau_{0}$. It seems improbable that a particular configuration could survive for long enough to reach a steady state in practice. A more plausible hypothesis is that the lifetime of a cell is of order $\tau_{0}$ and that the velocity $U \sim U_{0}$. These limitations must be a result of three-dimensional behaviour and may be related to Busse's oscillatory instability.

It is worth speculating briefly on the nature of convection between free boundaries. At $R=R_{c}$ convection sets in as rolls. For high Prandtl numbers the rolls should be stable throughout the viscous regime with $R / R_{c} \ll p^{\frac{3}{2}}$ (Straus 1972). At low Prandtl numbers the rolls develop oscillatory instabilities when the Reynolds number $R e \approx 6$ (Busse 1972). As the Rayleigh number increases these oscillations can grow in amplitude until the rolls break up into three-dimensional cells. However, the heat transport should not be drastically affected by this process, for rolls and cylinders have similar Nusselt numbers while experiments with rigid boundaries show only slight changes in $N$ as oscillations appear (Willis \& Deardorff 1970; Krishnamurti 1973). 
At very high Rayleigh numbers we expect that the interactions between adjacent three-dimensional cells would limit their lifetime to the turnover time $\tau_{0}$ and that the velocity would then be comparable with the free-fall velocity $U_{0}$. If fluid elements lose their excess heat while traversing a thermal boundary layer of thickness $\delta$ then, from (8),

and so

$$
\begin{gathered}
U_{0} d / \kappa \sim(d / \delta)^{2} \\
N \sim(d / \delta) \sim(p R)^{\frac{1}{4}} .
\end{gathered}
$$

The local Rayleigh number for the thermal boundary layer

$$
R_{\delta}=(\delta / d)^{3} R \sim\left(R / p^{3}\right)^{\frac{1}{2}}
$$

increases until the boundary layer itself becomes unstable. For a laminar boundary layer $R_{\delta} \sim R_{c}$ and so $N \sim R^{1}$. At low Prandtl numbers $R_{\delta}$ should be evaluated using a laminar conductivity and an eddy viscosity

$$
v_{e} \sim\left(g \alpha \Delta T \delta^{3}\right)^{\frac{1}{2}},
$$

based on the free-fall velocity through the boundary layer; it then follows that

$$
N \sim(p R)^{\frac{1}{3}}
$$

and the heat flux is independent of both $\nu$ and $d$ (Spiegel 1971 $a, b$ ). Finally, at extremely high Rayleigh numbers, shear-flow turbulence might increase the effective conductivity such that $N \sim(p R)^{\frac{1}{2}}$, as in ordinary mixing-length theory (Spiegel 1971 $a, b$ ).

This study has shown that the heat transport owing to steady laminar convection does not diminish with decreasing Prandtl number. Hence any bound on the Nusselt number must be independent of Prandtl number for finite $p$. Further progress requires fully three-dimensional time-dependent calculations. These might be expected to yield a Nusselt number that depends on the product $p R$ as $p \rightarrow 0$. Such an investigation could provide an understanding of turbulent astrophysical convection; it would also consume vastly more computer time than the work described here.

This research was supported by a grant from the Science Research Council and C. A. J. is grateful for an SRC Research Studentship. Computations were carried out on the IBM 370-165 of the University Computing Service. We wish to thank Dr D. O. Gough for encouraging us to proceed with this research and for countless comments and suggestions. We are also grateful for discussions with Professor G. K. Batchelor, Dr M. R. E. Proctor, Professor E. A. Spiegel and Professor J. S. Turner.

\section{Appendix. Numerical methods}

No account of numerical (or any other) experiments is complete without a description of the methods used sufficient to enable others to repeat the experiments and to gauge their accuracy. The computations discussed in $\S 4$ used a second-order finite-difference scheme on a staggered mesh, properly centred in 
space and time. All equations were expressed in conservative form, thereby avoiding any difficulties on the axis. This formulation is an extension to cylindrical co-ordinates of that developed by Roberts \& Weiss (1966), Moore et al. (1973) and Moore \& Weiss (1973) for a Cartesian mesh.

The system of nonlinear partial differential equations (8), (15) and (14) is solved by representing the variables $T, \Omega$ and $\psi$ on a square grid with spacing $\Delta r=\Delta z=h$. Let $r_{j}=j \Delta r, z_{k}=k \Delta z$ and $t^{n}=n \Delta t$, where $j=0,1, \ldots, N_{r}$ and $k=0,1, \ldots, N_{z}$, and put $T_{j, k}^{n}=T\left(r_{j}, z_{k}, t^{n}\right)$ etc. To represent the equations in conservative form we regard $T_{j, k}^{n}$ as the average value of $T$ over the box $\left\{r_{j-1}<r<r_{j+1}, z_{k-1}<z<z_{k+1}, 0<\phi<1\right\}$; similarly, the velocity $\mathbf{u}$ is the average flux across a surface and $\omega$ the average circulation round a surface, while $\psi$ is averaged trivially along a line element in the $\phi$ direction. The temperature equation is then averaged over the box, so that divergences are converted into fluxes, while the vorticity equation (12) is averaged over an area, with the curls transformed into circulations. The elliptic equation (14) can also be expressed in terms of circulations and is then solved implicitly by Fourier analysis in the $z$ direction. This procedure provides a unique difference formulation of the equations, which is more accurate than that obtained by simply expanding the derivatives, as has been shown by Weir (1976) for the analogous problem in a sphere.

In the temperature equation (8) the advective terms are treated by the leapfrog scheme which conserves $T$ exactly, and diffusion is represented by a DufortFrankel scheme which couples the independent meshes to each other. Then (8) becomes, for $j>1$,

$$
\begin{aligned}
\left(T_{j, k}^{n+1}-\right. & \left.T_{j, k}^{n}\right) \frac{4 r_{j} \Delta r \Delta z}{\Delta t}=-\left[\left\{(T u)_{j+1, k}^{n+\frac{1}{2}} r_{j+1}-(T u)_{j-1, k}^{n+\frac{1}{2}} r_{j-1}\right\} 2 \Delta z\right. \\
& \left.+\left\{(T w)_{j, k+1}^{n+\frac{1}{2}}-(T w)_{j, k-1}^{n+\frac{1}{2}}\right\} 2 r_{j} \Delta r\right]+4 \kappa\left[\frac{r_{j} \Delta r}{\Delta z}\left\{T_{j, k+1}^{n+\frac{1}{2}}-\left(T_{j, k}^{n}+T_{j, k}^{n+1}\right)+T_{j, k-1}^{n+\frac{1}{2}}\right\}\right. \\
& \left.+\frac{\Delta z}{\Delta r}\left\{r_{j+\frac{1}{2}} T_{j+1, k}^{n+\frac{1}{2}}-r_{j}\left(T_{j, k}^{n}+T_{j, k}^{n+1}\right)+r_{j-\frac{1}{2}} T_{j-1, k}^{n+\frac{1}{2}}\right\}\right]
\end{aligned}
$$

Hence we obtain the explicit difference equation

$$
\begin{aligned}
T_{j, k}^{n+1}= & T_{j, k}^{n}+\frac{\Delta t}{4\left(h^{2}+2 \kappa \Delta t\right)}\left[\frac { 1 } { r _ { j } } \left\{T_{j+1, k}^{n+\frac{1}{2}}\left(\psi_{j+1, k+1}^{n+\frac{1}{2}}-\psi_{j+1, k-1}^{n+\frac{1}{2}}\right)\right.\right. \\
& -T_{j-1, k}^{n+\frac{1}{2}}\left(\psi_{j-1, k+1}^{n+\frac{1}{2}}-\psi_{j-1, k-1}^{n+\frac{1}{2}}\right)-T_{j, k+1}^{n+\frac{1}{2}}\left(\psi_{j+1, k+1}^{n+\frac{1}{2}}-\psi_{j-1, k+1}^{n+\frac{1}{2}}\right) \\
& \left.+T_{j, k-1}^{n+\frac{1}{2}(}\left(\psi_{j+1, k-1}^{n+\frac{1}{2}}-\psi_{j-1, k-1}^{n+\frac{1}{2}}\right)\right\} \\
& \left.+4 \kappa\left\{T_{j, k+1}^{n+\frac{1}{2}}+T_{j, k-1}^{n+\frac{1}{2}}+\frac{r_{j+\frac{1}{2}}}{r_{j}} T_{j+1, k}^{n+\frac{1}{2}}+\frac{r_{j-\frac{1}{2}}}{r_{j}} T_{j-1, k}^{n+\frac{1}{2}}-4 T_{j, k}^{n}\right\}\right],
\end{aligned}
$$

where the velocities are expressed in terms of $\psi$ from (10). This holds for $j \geqslant 1$; for $j=0$ we must integrate (8) over the elementary cylinder

$$
\left\{0 \leqslant r<\Delta r, \quad z_{k-1}<z<z_{k+1}, \quad 0 \leqslant \phi \leqslant 2 \pi\right\},
$$


which differs topologically from the surrounding annuli. Integrating over the disk $\{0 \leqslant r<\Delta r\}$ we find that $w_{0, k}=2 \psi_{1, k} / h^{2}$ and so

$$
\begin{aligned}
\left.T_{0, k}^{n+1}=T_{0, k}^{n}+\frac{\Delta t}{\left(h^{2}+\right.}+3 \kappa \Delta t\right) & {\left[\frac { 1 } { h } \left\{T_{1, k}^{n+\frac{1}{2}}\left(\psi_{1, k+1}^{n+\frac{1}{2}}-\psi_{1, k-1}^{n+\frac{1}{2}}\right)-T_{0, k+1}^{n+\frac{1}{2}} \psi_{1, k+1}^{n+\frac{1}{2}}\right.\right.} \\
+ & \left.\left.T_{0, k-1}^{n+\frac{1}{2}} \psi_{1, k-1}^{n+\frac{1}{2}}\right\}+\kappa\left\{4 T_{1, k}^{n+\frac{1}{2}}+T_{0, k+1}^{n+\frac{1}{2}}+T_{0, k-1}^{n+\frac{1}{2}}-6 T_{0, k}^{n}\right\}\right] .
\end{aligned}
$$

The singularity at $r=0$ in (8), which is a property of the co-ordinate system and not of the physics, does not affect the finite-difference formulation.

Since (66) and (67) relate values of $T$ at times $t^{n}$ and $t^{n+1}$ to values at adjacent points at time $t^{n+\frac{1}{2}}$ it is not necessary to calculate and store $T$ at all points for all time levels. We therefore use a staggered mesh on which values of $T_{j, k}$ at points with $j+k$ even are stored at times $t^{n}, t^{n+1}, \ldots$, while those with $j+k$ odd are stored at times $t^{n+\frac{1}{2}}, t^{n+\frac{3}{2}}, \ldots$. This procedure halves the requirements for computer time and storage, with no appreciable loss of accuracy.

To find the potential vorticity $\Omega$ we should integrate the vorticity equation (12) over the area $\left\{r_{j-1}<r<r_{j+1}, z_{k-1}<z<z_{k+1}\right\}$. The result of this procedure is identical to that obtained by integrating the scalar equation (15) over a volume, whence we find, for $j \geqslant 1$, that

$$
\begin{aligned}
\Omega_{j, k}^{n+1}= & \Omega_{j, k}^{n}+\frac{\Delta t}{4 r_{j}\left[h^{2}+\nu \Delta t\left(2 r_{j}^{2}-\frac{1}{4} h^{2}\right) /\left(r_{j}^{2}-\frac{1}{4} h^{2}\right)\right]}\left[\Omega_{j+1, k}^{n+\frac{1}{2}}\left(\psi_{j+1, k+1}^{n+\frac{1}{2}}-\psi_{j+1, k-1}^{n+\frac{1}{2}}\right)\right. \\
& -\Omega_{j-1, k}^{n+\frac{1}{2}}\left(\psi_{j-1, k+1}^{n+\frac{1}{2}}-\psi_{j-1, k-1}^{n+\frac{1}{2}}\right)-\Omega_{j, k+1}^{n+\frac{1}{2}}\left(\psi_{j+1, k+1}^{n+\frac{1}{2}}-\psi_{j-1, k+1}^{n+\frac{1}{2}}\right) \\
& +\Omega_{j, k-1}^{n+\frac{1}{2}}\left(\psi_{j+1, k-1}^{n+\frac{1}{2}}-\psi_{j-1, k-1}^{n+\frac{1}{2}}\right)-2 g \alpha h\left(T_{j+1, k}^{n+\frac{1}{2}}-T_{j-1, k}^{n+\frac{1}{2}}\right) \\
& \left.+4 \nu\left\{r_{j}\left(\Omega_{j, k+1}^{n+\frac{1}{2}}+\Omega_{j, k-1}^{n+\frac{1}{2}}-\frac{2\left(2 r_{j}^{2}-\frac{1}{4} h^{2}\right)}{\left(r_{j}^{2}-\frac{1}{4} h^{2}\right)} \Omega_{j, k}^{n}\right)+\frac{r_{j+1}^{2}}{r_{j+\frac{1}{2}}} \Omega_{j+1, k}^{n+\frac{1}{2}}+\frac{r_{j-1}^{2}}{r_{j-\frac{1}{2}}} \Omega_{j-1, k}^{n-\frac{1}{2}}\right)\right] .
\end{aligned}
$$

The values of $\Omega$ on the axis (which are not actually needed for the computation) are obtained from the boundary condition (18):

$$
\Omega_{0, k}=\Omega_{2, k}
$$

to second order. Once again, it is only necessary to calculate $\Omega$ on a staggered mesh, at the same points as $T$.

The treatment of the elliptic equation (14) is somewhat more complicated, since $\psi$ and $\Omega$ are defined on a staggered mesh and we must therefore express the left-hand side in terms of $\psi_{j, k}$ and $\psi_{j \pm 1, k \pm \mathbf{1}}$. This can formally be done by integrating $\omega$ over the lozenge with vertices at $\left(r_{j \pm 1}, z_{k}\right)$ and $\left(r_{j}, z_{k \pm 1}\right)$ and then expressing the circulatory velocities around the perimeter of this lozenge as averages over the inclined plane surfaces, perpendicular to the $r, z$ plane, passing through $\left(r_{j-1}, z_{k+1}\right)$ and $\left(r_{j}, z_{k}\right)$ etc. Since $\mathbf{u}=\nabla \wedge\left(\psi r^{-1} \mathbf{e}_{\phi}\right)$ these averaged velocities can be expressed, through Stokes' theorem, in terms of $\psi_{j-1, k+1}, \psi_{j, k}$ etc. Once again, the result is identical to that obtained by integrating (14) over the 
volume formed by rotating the lozenge about the axis $r=0$. Using an obvious notation for difference operators, defined in Moore et al. (1973), we have

$$
\left(\begin{array}{lll}
\left(j-\frac{1}{2}\right)^{-1} & -4 j /\left(j^{2}-\frac{1}{4}\right) & \left(j+\frac{1}{2}\right)^{-1} \\
\left(j-\frac{1}{2}\right)^{-1} & \left(j+\frac{1}{2}\right)^{-1}
\end{array}\right) \psi_{j, k}=-2 j h^{4} \Omega_{j, k} .
$$

To solve this equation we first eliminate alternate columns, so as to leave only values of $\psi_{j, k}$ with $j$ even (Moore et al. 1973), and then expand $\psi$ in a truncated Fourier series in the $z$ direction, so that

$$
\psi\left(x_{j}, z\right)=\sum_{l=1}^{\frac{1}{2} N_{z}-1} \Psi_{j}^{l} \sin \frac{l \pi z}{d}
$$

The resulting equations have the form

$$
\frac{1}{j-1}(1+c) \Psi_{j-2}^{l}+\frac{j}{j^{2}-\frac{1}{4}}\left[\frac{\left(2 j^{2}-\frac{7}{2}\right)}{\left(j^{2}-1\right)}(1+c)-8\right] \Psi_{j}^{l}+\frac{1}{j+1}(1+c) \Psi_{j+2}^{l}=\Xi_{j}^{l},
$$

where $c=\cos (2 \pi l h / d)$ and

$$
\left(\begin{array}{ccc}
j-\frac{3}{2} & & j+\frac{3}{2} \\
& 4 j & \\
j-\frac{3}{2} & & j+\frac{3}{2}
\end{array}\right) \Omega_{j, k}=-\frac{1}{h^{4}} \sum_{l=1}^{\frac{1}{2} N_{z}-1} \Xi_{j}^{l} \sin \frac{l \pi z}{d} .
$$

They are solved by tridiagonal elimination and $\psi$ is synthesized from (71). Values of $\psi$ with $j$ odd are then given by

$$
\psi_{j, k}=\frac{\left(j^{2}-\frac{1}{4}\right)}{4 j}\left[\left(\begin{array}{ll}
\left(j-\frac{1}{2}\right)^{-1} & \left(j+\frac{1}{2}\right)^{-1} \\
\left(j-\frac{1}{2}\right)^{-1} & \left(j+\frac{1}{2}\right)^{-1}
\end{array}\right) \psi_{j, k}+2 h^{4} j \Omega_{j, k}\right] .
$$

Finally, since this procedure yields values of $\psi$ at the same points as $\Omega$ while (68) requires $\psi$ on the alternate mesh, it is necessary to interpolate for $\psi$. This is done using a fourth-order expression based on (14), so that

$$
\psi_{j, k}=\frac{j\left(j^{2}-\frac{1}{4}\right)}{\left(4 j^{2}-\frac{1}{2}\right)}\left[\left(\begin{array}{lll}
\left(j-\frac{1}{2}\right)^{-1} & j^{-1} & \left(j+\frac{1}{2}\right)^{-1}
\end{array}\right) \psi_{j, k}+\frac{1}{4} h^{4}\left(j-1 j^{-1} j+1\right) \Omega_{j, k}\right] .
$$

Solution of these equations proceeds very rapidly with fast Fourier transform techniques.

\section{Infinite Prandtl number}

When $p$ is infinite, (15) reduces to the linear elliptic equation

$$
\nabla \cdot\left[\frac{1}{r^{2}} \nabla\left(r^{2} \Omega\right)\right]=\left(\frac{g \alpha}{\nu}\right) \frac{1}{r} \frac{\partial T}{\partial r} .
$$

Happily, this has exactly the same form as (14). The vorticity can therefore be found by the same technique of Fourier analysis in the $z$ direction. Moreover, this process yields values of $\Omega$ at points adjacent to those where $T$ is defined; these values of $\Omega$ in turn yield $\psi$ at the same points, where it is needed for (66). No interpolation of $\psi$ or $\Omega$ is required and accuracy is correspondingly improved, as in Cartesian geometry (Moore et al. 1973; McKenzie, Roberts \& Weiss 1974). 


\section{Accuracy}

No detailed error analysis has been carried out but experience confirms that the accuracy of the cylindrical code is similar to that of the Cartesian code discussed in detail by Moore et al. (1973). Near the axis, where errors of order $(\Delta r / r)^{2}$ might appear significant, there were no signs of any irregular behaviour. The results were reliable (giving an error of less than $1 \%$ in $N$ ) provided that there were at least three intervals across the thermal boundary layers. The form of the cylindrical solutions allows more structure than two-dimensional rolls and the boundary layers are compressed near the axis. However, this affects only a small area and has little effect on global averages like the Nusselt number. Similarly, the descending plume at the outer boundary can become very thin, and must be adequately resolved.

We have implemented the schemes described above on meshes with $N_{z}=12$, 24 or 48 and $N_{r} \leqslant 84$. This has allowed us to follow convection with Nusselt numbers $N \lesssim 8$ with adequate accuracy and to obtain plausible results for $N \approx 10$. (The asymmetry between rising and sinking fluid prohibits the effective doubling of the mesh that was possible for two-dimensional rolls.) With Fourier analysis routines for $N_{z}=96$ it would be possible to obtain accurate solutions for $R \approx 500 R_{c}$.

With carefully constructed conservative difference schemes it is possible to compute highly nonlinear solutions with sufficient accuracy. For example, we attained an accuracy of $1 \%$ at $N \approx 5\left(R=20 R_{c}\right)$ with $N_{z}=24$. Liang et al. (1969), who expanded the derivatives before forming the difference equations, did not proceed beyond $N \approx 2$ with $N_{z}=29$. The availability of fast Fourier transforms makes the use of derived variables preferable to the method devised by Williams (1967) for investigating convection in an annulus. Other methods are reviewed by Orszag \& Israeli (1974). Although spectral and pseudo-spectral techniques allow considerably improved accuracy for a given amount of storage, finitedifference methods are comparable in terms of speed. They can also readily be extended to include, for example, the effects of a magnetic field.

\section{REFERENCES}

BAtCheLoR, G. K. 1956 J. Fluid Mech. 1, 177.

Busse, F. H. 1972 J. Flluid Mech. 52, 97.

ChandrasekHar, S. 1961 Hydrodynamic and Hydromagnetic Stability. Oxford: Clarendon Press.

Charlson, G. S. \& Sani, R. L. 1970 Int. J. Heat Mass Transfer, 13, 1479.

CharLson, G. S. \& SANI, R. L. 1971 Int. J. Heat Mass Transfer, 14, 2157.

Clever, R. M. \& Busse, F. H. 1974 J. Fluid Mech. 65, 625.

Gough, D. O., Spieged, E. A. \& Toomre, J. 1975 J. Fluid Mech. 68, 695.

JOSEPH, D. D. 1971 J. Fluid Mech. 47, 257.

Koschmieder, E. L. 1966 Beitr. Phys. Atmos. 39, 1.

Koschmidder, E. L. 1974 Adv. Chem. Phys. 26, 177.

Krishnamurti, R. 1973 J. Fluid Mech. 60, 285.

Liang, S. F., Vidal, A. \& Acrivos, A. 1969 J. Fluid Mech. 36, 239.

MoKenzie, D. P., Roberts, J. M. \& Weiss, N. O. 1974 J. Fluid Mech. 62, 465. 
Malkus, W. V. R. 1954 Proc. Roy. Soc. A 225, 196.

MaLkus, W. V. R. \& Veronis, G. 1958 J. Fluid Mech. 4, 225.

Moone, D. R., Peckover, R. S. \& Weiss, N. O. 1973 Comp. Phys. Comm. 6, 198.

Moore, D. R. \& Proctor, M. R. E. 1976 In preparation.

MOORE, D. R. \& WeISs, N. O. 1973 J. Fluid Mech. 58, 289.

Orszag, S. A. \& Israeli, M. 1974 Ann. Rev. Fluid Mech. 6, 281.

Peiclew, A. \& Southwelr, R. V. 1940 Proc. Roy. Soc. A 176, 312.

RAYLEIGH, LoRD 1916 Phil. Mag. 32 (6), 529.

Rogerts, K. V. \& WeIss, N. O. 1966 Math. Comp. 20, 272.

Schlüter, A., Lortz, D. \& Busse, F. 1965 J. Fluid Mech. 23, 129.

SPIEGEL, E. A. 1971 a Comm. Astrophys. Space Phys. 3, 53.

Spteger, E. A. $1971 b$ Ann. Rev. Astron. Astrophys. 9, 323.

Strats, J. M. 1972 J. Fluid Mech. 56, 353.

Threlfall, D. C. 1975 J. Fluid Mech. 67, 17.

WEIR, A. D. $1976 J$. Fluid Mech. (in press).

Werss, N. O. 1966 Proc. Roy. Soc. A 293, 310.

Wridiams, G. P. 1967 J. Atmos. Sci. 24, 144.

Willis, G. E. \& DeardorfF, J. W. 1970 J. F'luid Mech. 44, 661. 